\title{
Hybrid Ballistic-Diffusive Solution to the Frequency-Dependent Phonon Boltzmann Transport Equation
}

\section{Pareekshith Allu and Sandip Mazumder ${ }^{+}$}

Department of Mechanical and Aerospace Engineering

The Ohio State University,

Columbus, OH 43210, USA

Submitted to: International Journal of Heat and Mass Transfer, January 2016

${ }^{+}$Author for correspondence

Dr. Sandip Mazumder

Department of Mechanical and Aerospace Engineering

The Ohio State University

Suite E410, Scott Laboratory

201 West $19^{\text {th }}$ Avenue

Columbus, $\mathrm{OH} 43210$

E-mail: mazumder.2@osu.edu

Phone: (614) 247-8099

Fax: (614) 292-3163 


\begin{abstract}
The phonon Boltzmann Transport Equation (BTE) is appropriate for modeling heat conduction in semiconductor materials at the nanoscale. However, the BTE is difficult to solve on account of the directional and spectral nature of the phonon intensity, which necessitates angular and spectral discretization, and ultimately results in a large number (typically few hundreds) of four-dimensional partial differential equations. In the ballistic (large Knudsen number) regime, the phonon intensity is highly anisotropic, and therefore, angular resolution is desirable. However, in the diffusive (small Knudsen number) regime, the intensity is fairly isotropic, and hence, angular discretization is wasteful. In such scenarios, the method of spherical harmonics ( $\mathrm{P}_{\mathrm{N}}$ approximation) may be effectively used to reduce the large number of directional BTEs to a few partial differential equations. Since the Knudsen number is frequency dependent, the decision to preserve or eliminate angular discretization may be made frequency by frequency based on whether the spectral Knudsen number is large or small. In this article, a hybrid method is proposed in which for some frequency intervals (bands), full angular discretization is used, while for others, the $\mathrm{P}_{1}$ approximation is invoked to reduce the number of directional BTEs. The accuracy and efficiency of the hybrid method is tested by solving several steady state and transient nanoscale heat conduction problems in two and threedimensional geometries. Silicon is used as the candidate material. It is found that hybridization is effective in significantly improving the efficiency of solution of the BTE — sometimes by a factor of three — without significant penalty on the accuracy.
\end{abstract}

Keywords: Boltzmann Transport Equation; phonon; heat transfer; hybrid method; ballistic-diffusive; spectral Knudsen number 


\section{INTRODUCTION}

Effective thermal management is critical to further miniaturization and improvement of the power density and performance of next-generation electronic and optoelectronic devices. Typical feature sizes (or characteristic length scales) in such devices range between 10-1000 nm. In comparison, the dominant carriers of energy in semiconductor materials, namely phonons, have spectral mean free path of the order of a few hundred nanometers. For example, the mean free path of phonons in silicon at room temperature is approximately in the range $10-1000 \mathrm{~nm}$, with the mean around $300 \mathrm{~nm}$ [1]. Consequently, heat conduction in such devices cannot be described adequately using continuum equations, namely the Fourier law of heat conduction. The Boltzmann Transport Equation (BTE) for phonons serves as a viable alternative [2,3] for the prediction of nonequilibrium heat conduction in semiconductor materials at the nanoscale.

The BTE for phonons is an integro-differential equation with 7 independent variables: time, 3 spatial coordinates, 2 directional (or angular) coordinates, and frequency. Under the single-time relaxation approximation, the scattering term of the BTE can be significantly reduced (linearized) so that it becomes a 7-dimensional partial differential equation instead of an integro-differential equation. Nonetheless, even the linearized BTE is very challenging to solve because of its high dimensionality. Literature survey reveals that essentially three methods have been employed to date to numerically solve the phonon BTE: (a) the Monte Carlo method, (b) the lattice Boltzmann method, and (c) deterministic discretization-based methods. While the Monte Carlo method is suitable for the inclusion of complex physics such as dispersion, polarization, and various scattering mechanisms, as originally demonstrated by Mazumder and Majumdar [4], and 
subsequently used by other researchers [5,6], it is expensive for practical engineering applications, especially when high spatial resolution is sought. Recently, variance reduction techniques have enabled use of the Monte Carlo method for solution of the BTE in realistic three-dimensional structures [7]. The lattice Boltzmann method has only been used for the solution of the phonon BTE in simple two-dimensional structures $[8,9]$.

Deterministic solution of the phonon BTE based on discretization in all 7 dimensions has been brought to the limelight by Murthy and co-workers [10-13]. Such solutions make use of the discrete ordinates method and its variant, namely the control angle discrete ordinates method (referred to as the "finite-volume method for radiation" in the radiation literature $[14,15])$, for angular discretization. The control angle discrete ordinates method (CADOM) has been shown to be superior [14-16] since it mitigates ray effects inherent in the discrete ordinates method. Angular discretization of the 7dimensional BTE essentially results in a large number of 5-dimensional BTEs since the total solid angle of $4 \pi$ must be split into smaller solid angles by discretization of the independent polar and azimuthal angles. For example, Mittal and Mazumder [17,18] have shown that approximately 20 angles in each of the polar and azimuthal directions (resulting in a total of 400 solid angles) are necessary to obtain angular grid independent solutions for realistic three-dimensional geometries, particularly when the Knudsen number is large and transport is ballistic in nature. Other studies [19] have employed 64 angles for computations in two-dimensional (2D) geometries. It is the need for angular discretization - typically, at least several tens of solid angles - that renders deterministic solution of the BTE computationally challenging. For example, Ali et al. [20] have recently reported solutions to the transient, frequency-dependent phonon BTE in a 
device-like three-dimensional (3D) geometry discretized using 604,054 tetrahedral control volumes. They reported calculation times of about an hour for a single time-step on a 400-processor parallel machine.

In an effort to improve the computational efficiency for solution of the phonon BTE, several approaches have been explored. The vast majority of these approaches have attempted to utilize the physical attributes of phonon transport to propose a more efficient solution strategy. When the mean free path of a phonon is significantly larger than characteristic size of the system under consideration, its transport is so-called ballistic. In such a scenario, the Knudsen number, which is the ratio of the mean free path to the characteristic size of the system, is large, i.e., Kn $\square$ 1. Conversely, when the mean free path of the phonons is small in comparison to the characteristic size of the system, they undergo numerous scattering events, and transport is so-called diffusive. In such a scenario, $K n \square 1$. In the ballistic regime, transport is highly direction dependent. For example, the energy flux in a given direction (or intensity) may be very large along paths that directly connect hot and cold entities, while it may be small in other directions. Since scattering re-distributes energy directionally, abundant scattering, as is prevalent in the diffusive transport regime, makes the intensity more or less isotropic or direction independent. Based on the preceding discussion, it is fair to conclude that when scattering is rare, it is imperative that the directional nature of the intensity be captured well, while when scattering is dominant, this is not necessary. Consequently, the need for directional discretization of the BTE arises in ballistic regimes, and may be bypassed in diffusive regimes. This fundamental premise has been employed to develop hybrid solution strategies of different flavors for the BTE. One of the earliest hybrid solution strategies to 
the BTE was proposed by Chen and co-workers [21-23]. They based their method on the so-called Modified Differential Approximation (MDA) [24], originally developed for photon transport. In this approach, the phonon intensity is assumed to be a superposition of a ballistic intensity and a diffusive intensity. The diffusive intensity, by virtue of being isotropic, is determined by invoking the method of spherical harmonics. The resulting model is the so-called ballistic-diffusive equations (BDE) of phonon transport. In the BDE formulation [21-23], Chen and co-workers introduce artificial temperatures, namely "ballistic" and "media" temperatures. These temperatures do not have physical meaning and are introduced as mathematical artifacts. As a result, they make the formulation - in particular, the boundary conditions - difficult to understand and interpret. Also, the surface-to-surface exchange formulation used by Chen and co-workers [21-23] for determination of the ballistic component of the phonon intensity, which employs geometric viewfactors, is prohibitive for complex multi-dimensional geometries, in which case, determination of the viewfactors itself is a monumental task [25]. Mittal and Mazumder [17,18] developed an alternative formulation, also based on the MDA, which eliminates the need to use artificial temperatures. Furthermore, in their approach, the ballistic component of the phonon intensity is determined using the CADOM, making the solution algorithm amenable to large-scale computations in complex geometries. The method has been successfully demonstrated for the solution of the transient BTE in complex 3D nanostructures [18]. An important point to note is that both of these hybrid methods based on the MDA were demonstrated for the gray (frequency independent) BTE only, and important physics pertaining to phonons, namely dispersion and polarization effects, were not considered. 
Ballistic-diffusive hybridization of the frequency-dependent phonon BTE has also been pursued by researchers in the past. However, the philosophy followed in this regard is somewhat different. Based on the previously discussed premise that in the diffuse regime of phonon transport, the intensity is fairly isotropic, Loy et al. [26] proposed a strategy in which the Fourier law is directly used for frequencies for which the spectral Knudsen number is small, and the full BTE for frequencies for which the spectral Knudsen number is large. The model has been referred to as the hybrid Fourier-BTE model [26]. In this model, a cutoff Knudsen number is used to separate frequency intervals (bands) for which the full BTE is used versus bands for which the Fourier law is used. Clearly, use of the Fourier law as opposed to the full BTE for some bands has the potential to drastically reduce computational time. This study conclusively demonstrated the efficacy of cutoff Knudsen number based hybridization for efficient solution of the BTE. However, the model was developed only for steady state and for "small" changes in temperature. It was demonstrated only for regular 2D geometries with a structured Cartesian mesh.

In this article, we employ the afore-mentioned cutoff Knudsen number based hybridization philosophy to develop a general-purpose hybrid BTE solver that is valid for both steady state and transient calculations and for any temperature difference. Rather than use the Fourier law directly in the diffusive regime, the $\mathrm{P}_{1}$ approximation is invoked. This results in a second-order partial differential equation (in both space and time) similar in form to the Cattaneo equation. The boundary conditions for this equation are developed using the Marshak procedure [27]. In the ballistic spectral range, the intensity is determined by solving the full BTE using the CADOM method. The cutoff Knudsen 
number is first identified through a rigorous analysis of errors between analytical solution of the full BTE and the approximate (hybrid) method. The proposed hybrid ballisticdiffusive model includes relevant phonon physics, such as dispersion, polarization, variable (temperature and frequency dependent) relaxation time-scale, among others. It is demonstrated for both for 2D and 3D geometries discretized using unstructured mesh of arbitrary topology for both steady state and transient non-equilibrium heat conduction simulations.

\section{THEORY AND MATHEMATICAL FORMULATION}

Quantized lattice vibrations, known as phonons, are the dominant carriers of energy in semiconductor materials $[2,3]$. When the mean free path of the traveling phonons is of the same order of or larger than the characteristic dimension of the system under scrutiny, thermodynamic equilibrium ceases to exist. In such a scenario, the Fourier law of heat conduction is invalid, and the heat conduction is referred to as non-equilibrium heat conduction. The Boltzmann Transport Equation (BTE) for phonons is a proven mathematical model for describing non-equilibrium heat conduction in semiconductor materials.

\subsection{Boltzmann Transport Equation}

The BTE for phonons, under the single-time relaxation approximation, may be written as [2]

$\frac{\partial f}{\partial t}+\mathbf{v} \square \nabla f=\frac{f_{0}-f}{\tau}$ 
where $f$ is the distribution function of an ensemble of phonons, $f_{0}$ is the equilibrium number density function (i.e., the Bose-Einstein distribution function), $\tau$ is the overall relaxation time-scale of the phonons to relax to equilibrium due to all scattering processes in combination, and $\boldsymbol{v}$ is the group velocity. For an isotropic wavevector space, the distribution function, $f$, is a function of 8 independent variables, i.e., $f=f(t, \mathbf{r}, \hat{\mathbf{s}}, \omega, p)$, where $t$ is time, $p$ is polarization, and $\omega$ is the angular frequency. The space vector $\mathbf{r}$ has 3 components ( $x, y$, and $z$ in Cartesian coordinates), while the direction vector $\hat{\mathbf{s}}$ has 2 components, namely the polar angle $\theta$, and the azimuthal angle $\psi$. Since polarizations are discrete, it is customary to think of the distribution function as being a function of seven independent variables, with the implicit understanding that it is different for different polarizations. The Bose-Einstein distribution, $f_{0}$, on the other hand, is independent of direction and polarization, i.e., $f_{0}=f_{0}(t, \mathbf{r}, \omega)$. The group velocity, $\mathbf{v}$, is a function of direction, angular frequency, and polarization, i.e., $\boldsymbol{v}=\mathbf{v}(\hat{\mathbf{s}}, \omega, p)$, while the relaxation (or overall scattering) time-scale, $\tau$, is a function of angular frequency, temperature, and polarization i.e., $\tau=\tau(\omega, T, p)$. Equation (1) can be written in terms of the phonon intensity as follows [2,28]:

$$
\frac{\partial I_{\omega, p}}{\partial t}+\mathbf{v}_{\omega, p} \nabla \nabla I_{\omega, p}=\frac{\partial I_{\omega, p}}{\partial t}+\left|\mathbf{v}_{\omega, p}\right| \nabla \llbracket\left(I_{\omega, p} \hat{\mathbf{s}}\right)=\frac{I_{0, \omega, p}-I_{\omega, p}}{\tau_{\omega, p}},
$$

where $\boldsymbol{v}_{\omega, p}=\left|\mathbf{v}_{\omega, p}\right| \hat{\mathbf{s}}$, and the phonon intensities are defined as [28]

$$
\begin{aligned}
& I_{\omega, p}=I(t, \mathbf{r}, \hat{\mathbf{s}}, \omega, p)=\left|\mathbf{v}_{\omega, p}\right| \hbar \omega f D(\omega, p) / 4 \pi \\
& I_{0, \omega, p}=I_{0}(t, \mathbf{r}, \omega, p)=\left|\mathbf{v}_{\omega, p}\right| \hbar \omega f_{0} D(\omega, p) / 4 \pi
\end{aligned},
$$


where $I_{\omega, p}$ is the spectral directional phonon intensity, while $I_{0, \omega, p}$ is the equilibrium spectral phonon intensity. $D(\omega, p)$ is the phonon density of states per unit volume, and $\hbar$ is the Dirac constant, or reduced Planck constant. In order to emphasize the fact that in the frequency dependent BTE, the phonon intensity, relaxation time-scale and group velocity are functions of both frequency and polarization, the subscripts $\omega$ and $p$ have been introduced. In addition, since the phonon intensity is a function of direction, frequency, and polarization, Eq. (2) must be solved for all directions and frequencies, and summed over all directions, frequencies, and polarizations in order to compute the heat flux at any spatial location and instant of time, as is discussed next.

\subsection{Heat Flux, Divergence of Heat Flux, and Temperature}

The heat flux is related to the phonon intensity by the relationship [2,28]

$$
\mathbf{q}(t, \mathbf{r})=\sum_{p} \int_{\omega_{\min , p}}^{\omega_{\max , p}} \int_{4 \pi} I_{\omega, p}(t, \mathbf{r}, \hat{\mathbf{s}}, \omega, p) \hat{\mathbf{s}} d \Omega d \omega=\sum_{p} \int_{\omega_{\min , p}}^{\omega_{\max , p}} \mathbf{q}_{\omega, p}(t, \mathbf{r}) d \omega
$$

where the integrals are over all solid angles $(\Omega)$ and the frequency $(\omega)$ range of each

polarization. $\omega_{\max , p}$ and $\omega_{\min , p}$ are the maximum and minimum frequencies, respectively, corresponding to a given polarization, $p$. In Eq. (4), $\mathbf{q}_{\omega, p}$ denotes the spectral heat flux while $\mathbf{q}$ denotes the total heat flux at any instant of time, $t$, and location whose position vector is denoted by $\mathbf{r}$. Once the BTE [Eq. (2)] has been solved for all directions and frequencies, Eq. (4) must be used to compute the heat flux. While the solution of the BTE provides a mechanism to determine the heat flux, in order to determine the temperature distribution, one must apply the first law of thermodynamics. For a static medium, the first law may be written as [29] 
$\frac{\partial u}{\partial t}=-\nabla \sqsubset \mathbf{q}+\dot{q}_{g e n}$,

where $u$ is the internal energy per unit volume and $\dot{q}_{g e n}$ is the heat generation rate per unit volume due to other mechanisms, such as electron-phonon or photon-phonon interactions. The right hand side of Eq. (5) may be reduced further by making use of Eq. (4) and the BTE [Eq. (2)]. To do so, we first integrate Eq. (2) over the total solid angle $4 \pi$ to yield

$$
\frac{\partial}{\partial t}\left(\int_{4 \pi} I_{\omega, p} d \Omega\right)+\left|\mathbf{v}_{\omega, p}\right| \nabla\left(\int_{4 \pi} I_{\omega, p} \hat{\mathbf{s}} d \Omega\right)=\frac{1}{\tau_{\omega, p}}\left(4 \pi I_{0, \omega, p}-\int_{4 \pi} I_{\omega, p} d \Omega\right)
$$

Using the definition of the directionally integrated spectral intensity,

$$
G_{\omega, p}=\int_{4 \pi} I_{\omega, p} d \Omega
$$

and the spectral heat flux given by Eq. (4), Eq. (6) may be re-written as

$$
\frac{\partial G_{\omega, p}}{\partial t}+\left|\mathbf{v}_{\omega, p}\right| \nabla\left\lceil\mathbf{q}_{\omega, p}=\frac{1}{\tau_{\omega, p}}\left(4 \pi I_{0, \omega, p}-G_{\omega, p}\right)\right.
$$

Substitution of Eq. (8) into Eq. (5) yields

$$
\frac{\partial u}{\partial t}=-\sum_{p} \int_{\omega_{\min , p}}^{\omega_{\max , p}} \frac{1}{\left|\mathbf{v}_{\omega, p}\right|}\left[\frac{1}{\tau_{\omega, p}}\left(4 \pi I_{0, \omega, p}-G_{\omega, p}\right)-\frac{\partial G_{\omega, p}}{\partial t}\right] d \omega+\dot{q}_{g e n}
$$

The internal energy of a crystalline material at a given spatial location, $\mathbf{r}$, is related to the local temperature through the Bose-Einstein distribution and its density of state [2]

$$
u(\mathbf{r})=\sum_{p} \int_{\omega_{\min , p}}^{\omega_{\max , p}} u_{\omega, p} d \omega=\sum_{p} \int_{\omega_{\min , p}}^{\omega_{\max , p}} f_{0} \hbar \omega D(\omega, p) d \omega=\sum_{p} \int_{\omega_{\min , p}}^{\omega_{\max , p}} \frac{\hbar \omega D(\omega, p)}{\exp \left[\hbar \omega / k_{B} T(\mathbf{r})\right]-1} d \omega
$$

where the summation is over all polarization branches, and $k_{B}$ is the Boltzmann constant. $u_{\omega, p}$ denotes the contribution of phonons of frequency, $\omega$, and polarization, $p$, to the 
internal energy. Substitution of Eq. (10) into Eq. (9) yields a nonlinear equation in temperature, and the determination of the temperature field requires solution of this nonlinear equation. The temperature determined by this methodology is the local absolute temperature if local thermodynamic equilibrium exists. If the local number density distribution $(f)$ deviates from the equilibrium Bose-Einstein distribution $\left(f_{0}\right)$, the temperature computed using the above procedure is the so-called pseudo-temperature $[2,4]$.

\subsection{Non-Dimensional Form of the BTE}

As discussed in Section 1, the regime of phonon transport—ballistic versus diffusive — is delineated by the Knudsen number. The BTE encapsulates both regimes, and is therefore, appropriate for modeling quasi-ballistic (or ballistic-diffusive) transport. However, in Eq. (2), the Knudsen number is absent. Hence, the role of Knudsen number is not obviously evident. The Knudsen number and its role may be made more explicit by nondimensionalizing the BTE [Eq. (2)]. To do so, we first introduce the definition of the Knudsen number [2,3]:

$$
K n_{\omega, p}=\frac{\Lambda_{\omega, p}}{L}=\frac{\left|\boldsymbol{v}_{\omega, p}\right| \tau_{\omega, p}}{L}
$$

where $\Lambda_{\omega, p}$ is the mean free path of a phonon of a frequency $\omega$ and polarization $p$, and $L$ is the characteristic length scale. The mean free path is the product of the magnitude of the phonon group velocity (or speed) and the relaxation time-scale. Since the group velocity and the relaxation time-scale are both functions of frequency and polarization, it implies that the mean free path and the Knudsen number are also functions of frequency 
and polarization, as indicated by the superscripts in Eq. (11). In other words, phonons of different frequency will have different mean free paths and Knudsen numbers. Since the Knudsen number is frequency dependent, in the rest of this article, it will be referred to as the spectral Knudsen number.

The effect of the spectral Knudsen number is better delineated by first nondimensionalizing the BTE [Eq. (2)] using the following non-dimensional time and space variables:

$t^{*}=t / \tau_{\omega, p}, x^{*}=x / L, y^{*}=y / L, z^{*}=z / L$

Substitution of Eqs. (11) and (12) in Eq. (2) yields

$\frac{\partial I_{\omega, p}}{\partial t^{*}}+K n_{\omega, p} \nabla^{*} \sqsubset\left(I_{\omega, p} \hat{\mathbf{s}}\right)=I_{0, \omega, p}-I_{\omega, p}$

where $\nabla^{*} \equiv L \nabla$ is the non-dimensional gradient operator. Equation (13) clearly shows that if the spectral Knudsen number is small (diffusive regime), equilibrium will be restored, since the resulting solution to Eq. (13) at large times is $I_{0, \omega, p}=I_{\omega, p}$. Also, since $I_{0, \omega, p}$ is direction independent, the diffusive regime is characterized by a phonon intensity field that is isotropic. As indicated earlier, this finding is at the heart of the proposed hybrid formulation.

\section{4 $\quad \mathbf{P}_{1}$ Approximation}

Prior to invoking the $\mathrm{P}_{1}$ approximation, it is convenient to first write the BTE in terms of acoustic coordinates:

$x^{*}=\int_{0}^{x} \frac{d x^{\prime}}{\Lambda_{\omega, p}}, y^{*}=\int_{0}^{y} \frac{d y^{\prime}}{\Lambda_{\omega, p}}, z^{*}=\int_{0}^{z} \frac{d z^{\prime}}{\Lambda_{\omega, p}}$ 
The non-dimensional acoustic coordinates defined by Eq. (14) are very similar to those used in Eq. (12), except that the mean free path has been chosen as the characteristic length scale instead of a global length scale. Also, spatial variation of the mean free path (by virtue of the relaxation time-scale being a function of temperature) has been accommodated in this transformation. Substitution of the non-dimensional time given by Eq. (12), along with Eq. (14), into Eq. (2) yields the BTE written in acoustic coordinates:

$$
\frac{\partial \boldsymbol{I}_{\omega, p}}{\partial t^{*}}+\nabla^{*} \leftarrow\left(I_{\omega, p} \hat{\mathbf{s}}\right)=I_{0, \omega, p}-I_{\omega, p}
$$

where, following the definitions in Eq. (14), $\nabla^{*}$ is now the gradient operator in acoustic coordinates.

As shown in Section 2.3, in the diffuse (vanishingly small Knudsen number) limit, the phonon intensity is perfectly isotropic. Sufficiently close to the diffuse limit, wherein the intensity is more or less isotropic, it may be expressed by a linear function of direction - the so-called $\mathrm{P}_{1}$ approximation. Mathematically, the spectral intensity may be written as [27]

$$
I_{\omega, p}\left(t^{*}, \mathbf{r}^{*}, \hat{\mathbf{s}}, \omega, p\right)=J_{0, \omega, p}\left(t^{*}, \mathbf{r}^{*}, \omega, p\right)+\mathbf{J}_{1, \omega, p}\left(t^{*}, \mathbf{r}^{*}, \omega, p\right) \llbracket \hat{\mathbf{s}}
$$

where $J_{0}$ is a direction independent scalar coefficient, and $\mathbf{J}_{1}$ is a direction independent vector coefficient. The dot product with the direction vector renders the second term in Eq. (16) a scalar. Substitution of Eq. (16) into Eq. (15), followed by integration over the total solid angle $4 \pi$ and some algebraic manipulations yields

$$
\frac{\partial J_{0, \omega, p}}{\partial t^{*}}+\nabla^{*} \sqcap\left(\frac{1}{3} \mathbf{J}_{1, \omega, p}\right)=I_{0, \omega, p}-J_{0, \omega, p}
$$


Equation (17) has two unknowns, namely $J_{0, \omega, p}$ and $\mathbf{J}_{1, \omega, p}$, and cannot be solved. In order to derive an additional equation to close the system of equations, we multiply Eq. (17) by $\hat{\mathbf{s}}$ and then integrate the resulting equation over the total solid angle $4 \pi$. After tedious algebra, this yields

$\frac{\partial \mathbf{J}_{1, \omega, p}}{\partial t^{*}}+\nabla^{*} J_{0, \omega, p}=\mathbf{J}_{1, \omega, p}$

Equations (17) and (18) represent two equations with 2 unknowns: $J_{0, \omega, p}$ and $\mathbf{J}_{1, \omega, p}$. Unfortunately, since Eq. (18) is a vector equation, it is quite tedious to solve. In order to eliminate $\mathbf{J}_{1, \omega, p}$ in favor of $J_{0, \omega, p}$, we first differentiate Eq. (17) with respect to nondimensional time, yielding

$$
\frac{\partial^{2} J_{0, \omega, p}}{\partial t^{* 2}}+\frac{1}{3} \frac{\partial}{\partial t^{*}}\left[\nabla^{*} \square \mathbf{J}_{1, \omega, p}\right]=\frac{\partial I_{0, \omega, p}}{\partial t^{*}}-\frac{\partial J_{0, \omega, p}}{\partial t^{*}}
$$

Next, taking divergence of Eq. (18) yields

$$
\nabla^{*}\left(\frac{\partial \mathbf{J}_{1, \omega, p}}{\partial t^{*}}\right)+\nabla^{* 2} J_{0, \omega, p}=\nabla^{*} \square \mathbf{J}_{1, \omega, p}
$$

Finally, substituting Eqs. (20) and (17) into Eq. (19), following by rearrangement of the resulting equation, yields

$\frac{\partial^{2} J_{0, \omega, p}}{\partial t^{* 2}}+2 \frac{\partial J_{0, \omega, p}}{\partial t^{*}}-\frac{1}{3} \nabla^{* 2} J_{0, \omega, p}+J_{0, \omega, p}=I_{0, \omega, p}+\frac{\partial I_{0, \omega, p}}{\partial t^{*}}$

As indicated by Eq. (9), determination of temperature (or internal energy) requires determination of the spectral integrated intensity $G_{\omega, p}$. Substitution of Eq. (16) into Eq.

(7) yields

$$
G_{\omega, p}=\int_{4 \pi} I_{\omega, p} d \Omega=\int_{4 \pi}\left(J_{0, \omega, p}+\mathbf{J}_{1, \omega, p}\lceil\hat{\mathbf{S}}) d \Omega=4 \pi J_{0, \omega, p}\right.
$$


since $\int_{4 \pi} \hat{\mathbf{s}} d \Omega=0$. Thus, Eq. (21) may be multiplied by $4 \pi$ to yield a governing equation

for the integrated spectral intensity:

$$
\frac{\partial^{2} G_{\omega, p}}{\partial t^{* 2}}+2 \frac{\partial G_{\omega, p}}{\partial t^{*}}-\frac{1}{3} \nabla^{* 2} G_{\omega, p}+G_{\omega, p}=4 \pi\left(I_{0, \omega, p}+\frac{\partial I_{0, \omega, p}}{\partial t^{*}}\right)
$$

Transforming back to physical coordinates from acoustic coordinates yields

$$
\begin{aligned}
\tau_{\omega, p}^{2} \frac{\partial^{2} G_{\omega, p}}{\partial t^{2}}+2 \tau_{\omega, p} \frac{\partial G_{\omega, p}}{\partial t} & -\frac{1}{3} \tau_{\omega, p}\left|\mathbf{v}_{\omega, p}\right| \nabla\left\lfloor\left(\tau_{\omega, p}\left|\mathbf{v}_{\omega, p}\right| \nabla G_{\omega, p}\right)+G_{\omega, p}\right. \\
& =4 \pi\left(I_{0, \omega, p}+\tau_{\omega, p} \frac{\partial I_{0, \omega, p}}{\partial t}\right)
\end{aligned}
$$

Equation (24) is a linear partial differential equation that is second-order in both time and space. Therefore, it requires boundary conditions on all surfaces of the domain and two initial conditions. The boundary conditions for the full BTE [Eq. (2)] and the $\mathrm{P}_{1}$ approximation [Eq. (24)], are discussed next.

\subsection{Boundary Conditions}

For the original BTE [Eq. (2)], boundary conditions are necessary for the intensity. Two types of boundary conditions are generally used: (1) thermalizing, and (2) reflective.

A thermalizing boundary is analogous to a black surface for thermal radiation. It emits phonons based on the equilibrium energy distribution and absorbs any phonons that strike it, and is mathematically written as

$I_{\omega, p}\left(t, \mathbf{r}_{w}, \hat{\mathbf{s}}_{o}, \omega, p\right)=I_{0, \omega, p}\left(t, \mathbf{r}_{w}, \omega, p\right)$

where $\mathbf{r}_{w}$ is the position vector of the boundary or wall, and $\hat{\mathbf{s}}_{o}$ is the outgoing direction.

A reflective boundary condition is applied to adiabatic boundaries or symmetry planes. At such boundaries, the net normal heat flux is zero, which implies that the 
phonons are reflected without any loss of energy. The reflection of phonons can be diffuse, specular or partially specular. At a diffuse boundary, the incoming phonon intensity is split equally into all outgoing directions such that the net energy is conserved. Mathematically, this may be written as [27]

$$
I_{\omega, p}\left(t, \mathbf{r}_{w}, \hat{\mathbf{s}}_{o}, \omega\right)=\frac{1}{\pi} \int_{\hat{s}_{i}[\hat{\mathbf{n}}<0} I_{\omega, p}\left(t, \mathbf{r}_{w}, \hat{\mathbf{s}}_{i}, \omega\right) \hat{\mathbf{s}}_{i}\left[\hat{\mathbf{n}} d \Omega_{i}\right.
$$

where $\hat{\mathbf{s}}_{o}$ and $\hat{\mathbf{s}}_{i}$ are the outgoing and incoming directions, respectively. At a specular boundary, on the other hand, the incoming phonon intensity is diverted to the specular direction, which is given by [27]

$\hat{\mathbf{s}}_{o}=\hat{\mathbf{s}}_{s}=\hat{\mathbf{s}}_{i}-2\left|\hat{\mathbf{s}}_{i}\right| \hat{\mathbf{n}} \mid \hat{\mathbf{n}}$

where $\hat{\mathbf{s}}_{s}$ is the direction of specular reflection, and $\hat{\mathbf{n}}$ is the surface normal pointing outward from the boundary. For partially specular surfaces, a combination of the above two formulations is warranted, yielding

$$
I_{\omega, p}\left(t, \mathbf{r}_{w}, \hat{\mathbf{s}}_{o}, \omega\right)=\alpha I_{\omega, p}\left(t, \mathbf{r}_{w}, \hat{\mathbf{s}}_{s}, \omega\right)+\frac{1-\alpha}{\pi} \int_{\hat{s}_{i} \in \hat{\mathbf{n}}<0} I_{\omega, p}\left(t, \mathbf{r}_{w}, \hat{\mathbf{s}}_{i}, \omega\right) \hat{\mathbf{s}}_{i}\left[\hat{\mathbf{n}} d \Omega_{i}\right.
$$

where $\alpha$ is the so-called degree of specularity [4], and represents the fraction of the incoming energy that is reflected back in the specular direction.

In the case of the $\mathrm{P}_{1}$ approximation, the governing equation [Eq. (24)] is in terms of the integrated intensity, $G_{\omega, p}$, rather than the intensity itself. The boundary conditions for Eq. (24) may be developed by applying the so-called Marshak procedure [27], described next. The heat flux at any surface is the difference of the net outgoing flux and the net incoming flux. Since phonons do not change frequency upon interaction with the 
boundary, the preceding statement is also true on a spectral basis. At a thermalizing boundary, the spectral heat flux may be written in terms of the spectral intensity as

$$
\mathbf{q}_{\omega, p}\left[\hat{\mathbf{n}}=\int_{\hat{\mathbf{s}} \hat{\mathbf{n}}>0} I_{0, \omega, p} \hat{\mathbf{S}} \widehat{\mathbf{n}} d \Omega-\int_{\hat{\mathbf{s}} \hat{\mathbf{n}}<0} I_{\omega, p} \hat{\mathbf{S}}\left\lceil\hat{\mathbf{n}} d \Omega=\pi I_{0, \omega, p}-\int_{\hat{\mathbf{s}} \hat{\mathbf{n}}<0} I_{\omega, p} \hat{\mathbf{S}}\lceil\hat{\mathbf{n}} d \Omega\right.\right.
$$

Substitution of the $\mathrm{P}_{1}$ approximation for the intensity [Eq. (16)] into Eq. (29), followed by simplification yields

$\mathbf{q}_{\omega, p}\left\lceil\hat{\mathbf{n}}=2 \pi I_{0, \omega, p}-2 \pi J_{0, \omega, p}=2 \pi I_{0, \omega, p}-\frac{G_{\omega, p}}{2}\right.$

Substitution of Eqs. (4), (16) and (17) into Eq. (30), followed by several algebraic manipulations and transformation back to physical coordinates yields

$$
\tau_{\omega, p} \frac{\partial G_{\omega, p}}{\partial t}-\frac{2}{3} \tau_{\omega, p}\left|\mathbf{v}_{\omega, p}\right| \hat{\mathbf{n}} \backslash \nabla G_{\omega, p}=4 \pi I_{0, \omega, p}-G_{\omega, p}
$$

Equation (31) serves as the boundary condition for Eq. (24).

\subsection{Hybrid Method}

In the proposed hybrid method, a combination of the original full BTE, and the BTE after invoking the $\mathrm{P}_{1}$ approximation is used. For frequencies for which the spectral Knudsen number is above a prescribed cutoff value $\left(K n_{c}\right)$, the original BTE is used, while below that cutoff value, the $\mathrm{P}_{1}$ approximation is used. The prescribed cutoff Knudsen number is treated as a parameter so that its effect on accuracy and efficiency can be studied. The overall algorithm of the hybrid method may be summarized as follows:

Step 1: Based on the initial temperature distribution, an average temperature of the solution domain is computed. This average temperature is used to compute an average relaxation time scale, $\tau_{\omega, p}$, using prescribed relaxation time-scale expressions, such as 
the ones discussed in Section 4. The group velocity is next determined from the prescribed dispersion relationship and the spectral Knudsen number, $K n_{\omega, p}$, is computed using Eq. (11).

Step 2: For a given frequency, $\omega$, if the spectral Knudsen number is below the prescribed cutoff Knudsen number, i.e., $K n_{\omega, p}<K n_{c}$, then Eq. (24) is solved subject to boundary conditions given by Eq. (31). This provides $G_{\omega, p}$. For the same frequency, $\omega$, if the spectral Knudsen number is above the prescribed cutoff Knudsen number, i.e., $K n_{\omega, p}>K n_{c}$, then Eq. (2) is solved subject to boundary conditions given by Eqs. (25)(28). This provides $I_{\omega, p}$. In this case, Eq. (7) is to be used to compute $G_{\omega, p}$ from $I_{\omega, p}$.

Step 3: The $G_{\omega, p}$ obtained in Step 2 for all frequencies is substituted into Eq. (9) to compute the temperature of the domain. The spectral heat flux can be computed using Eq. (4) for $K n_{\omega, p}>K n_{c}$, and Eq. (30) for $K n_{\omega, p}<K n_{c}$.

Step 4: The average temperature of the domain is re-computed and Steps 1-3 are repeated until convergence.

In the hybrid method, just described, the split between the two types of equations (full BTE versus $\mathrm{P}_{1}$ ) is done based on an average (reference) temperature value and an average relaxation time-scale. However, this does not imply that that the method does not permit use of spatially varying relaxation time scales, as is evident from both Eq. (2) and Eq. (24). The overall solution algorithm of the hybrid method is depicted schematically in Fig. 1. In the section to follow, the numerical procedure employed to solve the full BTE [Eq. (2)] and the $\mathrm{P}_{1}$ equation [Eq. (24)] is discussed. 


\section{NUMERICAL PROCEDURE}

The full BTE, namely Eq. (2), is spatially discretized using the unstructured finitevolume procedure [30]. For angular discretization, the CADOM [14,15] is used because it is known to mitigate so-called ray effects [14-16]. In the CADOM, the total solid angle, $4 \pi$, is discretized using pre-determined finite solid angles (or control angles), each centered along direction $\hat{\mathbf{s}}_{i}$, defined by [27] $\hat{\mathbf{s}}_{i}=\sin \theta_{i} \sin \psi_{i} \hat{\mathbf{i}}+\sin \theta_{i} \cos \psi_{i} \hat{\mathbf{j}}+\cos \theta_{i} \hat{\mathbf{k}}$, where $\theta_{i}$ and $\psi_{i}$ are polar and azimuthal angles, respectively, that define the discrete direction $i$. The BTE, after spatial, spectral, and angular discretization, may be written as [20]:

$$
\frac{\partial I_{i, j, p, k}}{\partial t} V_{k} \Omega_{i}+|\boldsymbol{v}|_{j, p, k} \sum_{f} I_{i, j, p, f} \mathbf{S}_{i}\left[\hat{\mathbf{n}}_{f} A_{f}=\frac{1}{\tau_{j, p, k}}\left(I_{0, j, p, k}-I_{i, j, p, k}\right) V_{k} \Omega_{i},\right.
$$

where the indices span the following values: $i=1,2, \ldots, N_{\text {dir }}, j=N_{\text {band, Kn }<K_{c}}+1, \ldots, N_{\text {band }}$, $k=1,2, \ldots, N_{\text {cell }}$, and $p \in \mathrm{LA}, \mathrm{TA}, \mathrm{LO}, \mathrm{TO}$. The quantity $N_{b a n d, K n<K n_{c}}$ denotes the number of bands for which the Knudsen number is below the cutoff Knudsen number. In Eq. (32), $I_{i, j, p, k}$ denotes the phonon intensity along the $i$-th direction (or solid angle), $j$-th spectral interval (or band), $p$-th polarization, and $k$-th cell (or control volume). The total number of directions (discrete solid angles) is denoted by $N_{\text {dir }}$, the total number of bands by $N_{\text {band }}$, and the total number of cells by $N_{\text {cell }}$. $V_{k}$ denotes the volume of the $k$-th cell. $\Omega_{i}$ denotes the solid angle corresponding to the $i$-th direction, and is defined as

$$
\Omega_{i}=\int_{\Delta \theta_{i} \Delta \psi_{i}} \sin \theta d \theta d \psi=2 \sin \theta_{i} \sin \left(\frac{\Delta \theta_{i}}{2}\right) \Delta \psi_{i}
$$


The equilibrium intensity of the $j$-th band, $p$-th polarization and $k$-th cell is denoted by $I_{0, j, p, k}$. The quantity, $\mathbf{S}_{i}$, in Eq. (13) is defined as follows:

$\mathbf{S}_{i}=\int_{\Delta \theta_{i}} \int_{\Delta \psi_{i}} \hat{\mathbf{s}}_{i} \sin \theta d \theta d \psi$

The intensity at the face, denoted by $I_{i, j, p, f}$, is an unknown in Eq. (32), along with the cell-center intensity, $I_{i, j, p, k}$. The intensity at the face needs to be expressed in terms of the cell-center values prior to solution of Eq. (32). The face intensity is generally approximated as the upwind cell's intensity $[14,15]$, i.e.,

$I_{i, j, p, f}= \begin{cases}I_{i, j, p, k} & \text { if } \quad \hat{\mathbf{s}}_{i}\left[\hat{\mathbf{n}}_{f}>0\right. \\ I_{i, j, p, N} & \text { if } \quad \hat{\mathbf{s}}_{i}\left[\hat{\mathbf{n}}_{f}<0\right.\end{cases}$

where $N$ denotes the neighbor to the $k$-th cell, and $\hat{\mathbf{n}}_{f}$ is the outward-pointing surface normal to face $f$ from the perspective of cell $k$, as shown in Fig. 2.

The $\mathrm{P}_{1}$ equation [Eq. (24)], on the other hand, does not require angular discretization. It requires only spatial discretization, and this is accomplished using the unstructured finite volume procedure [30]. The resulting discrete $\mathrm{P}_{1}$ equation is written as

$$
\begin{aligned}
\tau_{j, p . k}^{2} \frac{\partial^{2} G_{j, p, k}}{\partial t^{2}} V_{k}+2 \tau_{j, p, k} \frac{\partial G_{j, p, k}}{\partial t} V_{k} & -\frac{\tau_{j, p, k}\left|\mathbf{v}_{j, p}\right|^{2}}{3} \sum_{f} \tau_{j, p, f}\left(\hat{\mathbf{n}}_{f} \square \nabla G_{j, p, f}\right) A_{f} \\
& +G_{j, p, k} V_{k}=4 \pi\left(I_{0, j, p, k}+\tau_{j, p, k} \frac{\partial I_{0, j, p, k}}{\partial t}\right) V_{k}
\end{aligned}
$$

where the indices span the following values: $j=1,2, \ldots, N_{b a n d, K n<K n_{c}}, k=1,2, \ldots, N_{\text {cell }}$, and $p \in \mathrm{LA}, \mathrm{TA}, \mathrm{LO}, \mathrm{TO}$. In this case, it is necessary to express the normal flux at each face, $\hat{\mathbf{n}}_{f} \square \nabla G_{j, p, f}$, in terms of the cell-center values, $G_{j, p . k}$, prior to solution of Eq. (36). The 
detailed mathematical procedure to do this may be found elsewhere [30], and is omitted here for the sake of brevity.

In the context of solution of Eq. (32), for each band $j$, polarization $p$, and direction $i$, Eq. (32) represents a linear system of algebraic equations with $N_{\text {cell }}$ unknowns. In order to completely solve Eq. (32), $\quad N_{d i r} \times N_{b a n d}$ such linear systems must be solved. In the present study, the linear systems were solved using the Generalized Minimal Residual (GMRES) solver [31] after incomplete LU (ILU) pre-conditioning. 20 Krylov sub-spaces were used, and 6 orders of magnitude reduction of residual were enforced for the GMRES solver. Likewise, Eq. (36) was solved for each band using the same linear algebraic equation solver.

\section{RESULTS AND DISCUSSION}

\subsection{Cutoff Knudsen Number}

One of the important input parameters in the proposed hybrid method is the cutoff Knudsen number. What is an appropriate value for this quantity? To answer this question, the gray steady state BTE was solved in a one-dimensional (1D) plane parallel geometry, and the Knudsen number was treated as a parameter. The top and bottom boundaries were assumed to be isothermal with a difference of $10 \mathrm{~K}$ between the two temperatures. For this particular scenario, the BTE, when coupled with the energy equation, results in a Fredholm integral equation [27] that may be solved analytically to determine the temperature distribution, as well as the flux at the two boundaries. This solution is free of any numerical errors (as would occur if the CADOM were to be used) and can be considered an appropriate benchmark solution. The BTE, subject to the $\mathrm{P}_{1}$ approximation, 
was also solved for the same scenario. The governing $\mathrm{P}_{1}$ equation [Eq. (36)] reduces to a second-order linear ODE in this case, which may also be solved analytically. Thus, a comparison of the solutions produced by the two methods isolates the error incurred by the $\mathrm{P}_{1}$ approximation, as opposed to any numerical error. Table 1 shows the results computed using the BTE and the BTE with the $\mathrm{P}_{1}$ approximation. Since the problem under consideration is a $1 \mathrm{D}$ steady state problem, the heat fluxes at the two boundaries are equal by energy conservation. Hence, only one of those fluxes, and the error associated with using the $\mathrm{P}_{1}$ approximation is reported in Table 1 for a large range of Knudsen numbers. Also reported in the last column is the percentage $\mathrm{L}^{2}$ Norm of the error in the spatial temperature distribution computed using the $\mathrm{P}_{1}$ approximation. The nondimensional flux reported in Table 1 is the ratio of the actual flux and the maximum possible flux, $\sigma_{P}\left(T_{H}^{4}-T_{C}^{4}\right)$, where $\sigma_{P}$ is the Stefan-Boltzmann constant for phonons (=186.63 W/m $\left./ \mathrm{m}^{2} / \mathrm{K}^{4}\right)[2,28]$, and $T_{H}$ and $T_{C}$ are the absolute temperatures of the hot and cold boundaries, respectively. Table 1 shows that at small Knudsen numbers, as contended earlier, the $\mathrm{P}_{1}$ approximation reproduces the solution of the exact BTE. At large Knudsen numbers, the Casimir limit is manifested, and the exact analytical solution produces a limiting non-dimensional flux of unity. The heat flux predicted by the $\mathrm{P}_{1}$ approximation may be computed by combining the steady-state gray renditions of Eqs. (9), (24), (30) and (31), to yield $\frac{q}{\sigma_{p}\left(T_{H}^{4}-T_{C}^{4}\right)}=\frac{1}{\frac{3}{4 K n}+1}$. It is clear from the preceding expression that as the Knudsen number tends to infinity, the non-dimensional heat flux predicted by the $\mathrm{P}_{1}$ approximation also tends to unity. It is in the intermediately high Knudsen number range that the error between the exact BTE and the $\mathrm{P}_{1}$ approximation is 
the largest. Below a Knudsen number is of 0.1 , the error in the heat flux is less than $1 \%$. Based on this finding, in the remainder of this work, a nominal cutoff Knudsen number of 0.1 is used for the hybrid method.

\subsection{Steady State Analysis}

In this section, results of steady state analysis of a $2 \mathrm{D}$ sub-micron heat conduction problem that has been considered in previous studies [17,18,23] are reported. This particular problem is analyzed in-depth to identify the pros and cons of the hybrid method both in terms of accuracy and computational efficiency. Toward the end of this section, results for a $3 \mathrm{D}$ problem are also reported to demonstrate the feasibility of the hybrid method for large-scale computations.

The problem under consideration is comprised of a square 2D block of silicon with a heating element at the bottom, as shown in Fig. 3 . The square block is of size $1 \mu \mathrm{m}$ x $1 \mu \mathrm{m}$, while heating element is of size $0.1 \mu \mathrm{m}$. The heating element is maintained at a constant temperature $T_{H}$, while all other boundaries are held at a fixed temperature $T_{C}(<$ $T_{H}$ ). For the first set of calculations, $\Delta T=T_{H}-T_{C}=10 \mathrm{~K}$ was used. In order to alter the Knudsen number, the following three temperature ranges were considered:

Case 1: $T_{H}=205 \mathrm{~K}, T_{C}=195 \mathrm{~K}$

Case 2: $T_{H}=255 \mathrm{~K}, T_{C}=245 \mathrm{~K}$

Case 3: $T_{H}=305 \mathrm{~K}, T_{C}=295 \mathrm{~K}$ 
The time-scales for Normal and Umklapp scattering in silicon were obtained from the literature [32], and are given by:

$\tau^{-1}=B_{L} \omega^{2} T^{3}$ (Normal and Umklapp, LA),

$\tau^{-1}=B_{T N} \omega T^{4}$ (Normal, TA),

$\tau^{-1}=B_{T U} \omega^{2} \sinh \left(\hbar \omega / k_{B} T\right)($ Umklapp, TA $)$

where the constants in Eq. (37) are as follows: $B_{L}=2 \times 10^{-24} \mathrm{~K}^{-3} \mathrm{rad}^{-2} \mathrm{~s}, B_{T N}=9.3 \times 10^{-13}$ $\mathrm{K}^{-4} \mathrm{rad}^{-1}, B_{T U}=5.5 \times 10^{-18} \mathrm{rad}^{-2} \mathrm{~s}$. The Mathiessen rule [2] was then used to compute the overall relaxation time-scale. Optical phonons were not considered in this study because their contributions to thermal transport in silicon have been shown to be marginal [6] except at high temperature. Dispersion relationships for silicon were used from Brockhouse and Dolling [33], which were then used to compute the phonon group velocities for all frequencies and polarizations. Using the relaxation time-scale and the group velocities as inputs, the spectral Knudsen numbers may be computed using Eq. (11). Figure 4 shows the spectral Knudsen numbers at two different temperatures for both longitudinal acoustic (LA) and transverse acoustic (TA) phonons. It is evident that the spectral Knudsen number range shifts to higher values at lower temperatures, indicating a shift toward ballistic transport from diffusive transport as temperature is decreased.

First, in order to quantify the errors, steady state computations were performed for the lowest temperature case (Case 1) using three different methods: (1) the original full BTE solved using the CADOM method-henceforth referred to as "CADOM," (2) the BTE under the $\mathrm{P}_{1}$ approximation - henceforth referred to as "P1," and (3) the hybrid method-henceforth referred to as "HYBRID." The low-temperature case has the highest degree of non-equilibrium, and hence, was deemed most appropriate for quantifying the 
errors. The frequency space was discretized using 40 spectral intervals (or bands). 20 equally spaced bands were used between 0 and $\omega_{\max , \mathrm{TA}}$, and 20 additional equally spaced bands were used between $\omega_{\max , \mathrm{TA}}$ and $\omega_{\max , \mathrm{LA}}$. The number of bands were selected based on the findings of previous studies [6,20]. A total of 100 directions were used for angular discretization. Both structured and unstructured mesh topologies were explored: a $100 \mathrm{x}$ 100 structured mesh, as well as an unstructured mesh with 22,896 triangular cells. Both meshes yielded grid-independent solutions, and almost identical results. Here, only results computed on the structured mesh are reported. Figure 5 shows the nondimensional heat flux $\left(=\mathbf{q}\left[\hat{\mathbf{n}} / \pi\left(I_{0, H}-I_{0, C}\right)\right)\right.$ distributions at the top and bottom walls. For these results, a cutoff Knudsen number, $K n_{c}=0.1$, was used. It is clear that the $\mathrm{P}_{1}$ approximation is inaccurate for this Knudsen number range. In fact, it yields unphysical results at the bottom wall, as indicated by non-dimensional fluxes exceeding unity. The hybrid method, on the other hand, yields physically meaningful results that are much closer to those produced by the full BTE (CADOM). The average errors for the hybrid method are $13.5 \%$ and $1.53 \%$ at the bottom and top walls, respectively. The absolute errors in the centerline temperature (going from the bottom to the top wall along the center of the heating element) are shown in Fig. 6. Once again, it is seen that the hybrid method yields a maximum error of $0.1 \mathrm{~K}$ (out of $10 \mathrm{~K}$ ), while the $\mathrm{P}_{1}$ method yields a maximum error of $0.42 \mathrm{~K}$. It is worth pointing out that irrespective of the global Knudsen number (based on a global characteristic length scale), at the boundaries, the intensity is always anisotropic, and hence, the $\mathrm{P}_{1}$ approximation will always yield inaccurate solutions close to the boundaries, as is evident from Fig. 6. Peraud and Hadjiconstantinou [34] perform asymptotic analysis and match an inner (close to boundaries) solution 
derived from the BTE with an outer (away from boundaries) solution derived from a diffuse approximation. Similar trends were observed for the other two cases, with the notable exception that for the hybrid method, the magnitude of the errors decreased as the temperature was increased, while for the $\mathrm{P}_{1}$ method, the errors decreased for the bottom wall but increased for the top wall. A summary of the errors incurred by the two methods for all 3 cases is presented in Table 2. The effect of the cutoff Knudsen number on the accuracy of predictions of the hybrid method is also reported in Table 2. As expected, a lower cutoff Knudsen number yielded more accurate results in the quasi-ballistic regime (Case 1), while no noticeable improvement was manifested closer to the diffusive regime (Case 3). This may be attributable to the fact that closer to the diffusive regime, the inaccuracy of the hybrid method is dictated by the inherent inaccuracy of the $\mathrm{P}_{1}$ approximation, which is used for the majority of bands at high temperature. Overall, the results conclusively show that the hybrid method is more accurate than the $\mathrm{P}_{1}$ method, and it appears that a nominal cutoff Knudsen number value of 0.1 is an appropriate choice.

The improved accuracy of the hybrid method comes at the cost of increased computational time, as shown in Table 2. All computations were performed on a 2.33 GHz Intel Core 2 Duo processor. Close to the ballistic limit (Case 1), the hybrid method is only slightly more efficient than solving the full BTE using CADOM. However, close to the diffusive limit (Case 3), the hybrid method is significantly more efficient-by almost a factor of 3 - compared to the full BTE. Overall, the hybrid method appears to be a reasonable compromise between accuracy and efficiency. 
The hybrid method was also tested for a 3D geometry, depicted in Fig. 7(a). The test case is comprised of a block of silicon of dimensions $1 \mu \mathrm{m} \times 1 \mu \mathrm{m} \times 0.3 \mu \mathrm{m}$, with a circular heater on the top surface of radius $0.05 \mu \mathrm{m}$. The operating conditions are the same as Case 1. The geometry was discretized using 21,978 tetrahedral cells. The frequency space was discretized into 40 bands and a total of 100 solid angles were used for angular discretization. The steady state temperature contours obtained using the three methods are depicted in Fig. 7(b)-(d). The mesh employed for these calculations did not yield grid independent solutions. Further refinement would require parallelization of the hybrid solver, which is left to future work. Although the differences in the solutions produced by the three methods are not easily decipherable from Fig. 7, these calculations demonstrate that the proposed hybrid method is scalable to computations in 3D geometries.

\subsection{Transient Analysis}

As mentioned in Section 1, hybrid methodologies for the phonon BTE that have been proposed by other researchers have only been demonstrated for steady state calculations. In this section, we assess the efficacy of our hybrid method for transient quasi-ballistic heat conduction situations. The geometry, mesh, and operating conditions considered for this study are the same as those described for Case 1 in Section 4.1. For time advancement, the backward Euler (or implicit) method [30] was used with a time-step size of $d t=\tau_{\max } / 100$, where $\tau_{\max }$ corresponds to the largest spectral relaxation timescale. This results in $\Delta t=0.16 \mathrm{~ns}$. While this time step size may appear somewhat large compared to time step sizes used in Monte Carlo methods, this is possible because the 
time integration scheme used here is fully implicit. On the other hand sub-dividing the relaxation time-scale into 100 intervals allows adequate resolution of the scattering processes. For these computations, the nominal cutoff Knudsen number of 0.1 was used. Figure 8 shows a comparison of the heat fluxes computed using the three methods. The $\mathrm{P}_{1}$ method is found to be grossly inaccurate both for the top and bottom walls at small times, while its accuracy for the top wall improves as time proceeds. In contrast, the accuracy of the hybrid method is consistently good and does not change significantly with time, indicating the reliability of the method for transient simulations. The oscillations in the top wall heat fluxes at small times are a manifestation of ray effects in the CADOM, which mitigates this effect in comparison to the standard discrete ordinates method, but cannot eliminate it altogether. Figure 9 illustrates the temporal evolution of the temperature distribution computed using the hybrid method. A large temperature slip is observed near the heating surface, which is a telltale sign of non-equilibrium quasiballistic transport. The total CPU time taken to conduct the calculations for 100 time steps were found to be 45.4 hours for CADOM, 3.5 hours for P1 and 37.7 hours for the Hybrid method. These computational times are consistent (proportional) with the times taken for steady state calculations by the three methods (Table 2). Hence, the same conclusions as steady state calculations may be drawn for the relative efficiency of the three methods for transient calculations.

\section{SUMMARY AND CONCLUSIONS}

The BTE for phonons is appropriate for modeling phonon-mediated non-equilibrium transport of heat in semiconductor materials. Unfortunately, since it is a seven- 
dimensional equation, its solution is challenging. In particular, the directional nature of the BTE renders its solution computationally extremely time-consuming since several tens of directional BTEs need to be solved once the total solid angle has been discretized. Traditionally, the discrete ordinates method or the control angle discrete ordinates method (CADOM) has been used for angular discretization of the BTE. Two methods are explored in this study with the goal of reducing computational time without significant penalty on accuracy. The first of these is the lowest order spherical harmonics method, namely the $\mathrm{P}_{1}$ approximation. The second approach combines the $\mathrm{P}_{1}$ approximation with the CADOM method, resulting in the so-called hybrid method. The philosophy in both methods is to reduce the number of directional BTEs that need to be solved as a means to reduce computational time.

In the $\mathrm{P}_{1}$ method, irrespective of the regime of transport (ballistic or diffusive), the phonon intensity is assumed to vary linearly with direction. Under this approximation, the direction BTEs may be reduced to a single second-order unsteady Helmholtz PDE with Robin boundary conditions. Its solution is extremely efficient compared to the several tens of directional BTEs. For the cases studied here, the computational efficiency improved significantly with speed-up factors as large as 10. Unfortunately, in the strongly ballistic regime (large Knudsen number), the $\mathrm{P}_{1}$ approximation was found to yield grossly inaccurate results, and in some cases, results that are unphysical. Based on the findings of this study, it may be concluded that the $\mathrm{P}_{1}$ approximation may only be used in scenarios where the entire range of spectral Knudsen numbers is below approximately 0.1. Such a situation may arise at high temperatures or for systems that are relatively large. A similar conclusion has been reached by Peraud and Hadjiconstantinou [34] in their recent study. 
It should be pointed out that despite its limitations, the $\mathrm{P}_{1}$ approximation is still capable of accurately modeling weakly ballistic transport, and as opposed to the Fourier law, can still account for the spectral nature of phonon transport.

In an effort to improve the accuracy of the $\mathrm{P}_{1}$ approximation, a hybrid method was proposed. In the hybrid method, the $\mathrm{P}_{1}$ approximation is only used in spectral regions where the Knudsen number is below a prescribed cutoff Knudsen number. In the remaining spectral region (above the cutoff Knudsen number), the original BTE is solved with full angular discretization. The hybrid method defaults to the $\mathrm{P}_{1}$ approximation if the spectral Knudsen numbers are all below the cutoff value, and defaults to the original BTE if the spectral Knudsen numbers are above the cutoff value. Through rigorous analysis, it was found that a value of 0.1 is appropriate for the cutoff Knudsen number. With this nominal value for the cutoff Knudsen number, the hybrid method was explored for a variety of steady state and transient non-equilibrium heat conduction problems, and in all cases, its accuracy was found to be reasonable, with maximum local errors never exceeding $15 \%$, and average local errors of less than $5 \%$ for both heat flux and temperature. On the other hand, the computational efficiency improved significantly in comparison to the solution of the original BTE with speed-up factors ranging between 1.2 and 3. Overall, the hybrid method is a good compromise between accuracy and efficiency and automatically adapts itself to the problem at hand (transport regime).

\section{ACKNOWLEDGMENTS}

Financial support for this work was provided, in part, by the National Science Foundation's Computation and Data-Enabled Science and Engineering (CDESE) 
Program through Award Number 1250215. ESI Group is acknowledged for providing free licenses to CFD-ACE+ ${ }^{\mathrm{TM}}$. 


\section{REFERENCES}

[1] Ju, Y.S., and , Goodson, K. E., (1999), "Phonon Scattering in Silicon Films with Thickness of Order 100 nm,'” Appl. Phys. Lett., 74, No. 20, pp. 3005-3007, DOI: 10.1063/1.123994

[2] Tien, C.L., Majumdar, A., and Gerner, F.M., eds., 1998, Microscale Energy Transport, Taylor and Francis.

[3] Zhang, Z., (2007), Nano/Microscale Heat Transfer, McGraw Hill, New York.

[4] Mazumder, S., and Majumdar, A., (2001), "Monte Carlo Study of Phonon Transport in Solid Thin Films Including Dispersion and Polarization," Journal of Heat Transfer, Vol. 123, pp. 749-759, DOI: 10.1115/1.1377018

[5] Lacroix, D., Joulain, K., and Lemonnier, D. (2005), "Monte Carlo transient phonon transport in Silicon and Germanium at nanoscale," Phys. Rev. B, Vol. 72, 064305(111), DOI: 10.1103/PhysRevB.72.064305

[6] Mittal, A., and Mazumder, S., (2010), "Monte Carlo Study of Phonon Heat Conduction in Silicon Thin Films Including Contributions of Optical Phonons," Journal of Heat Transfer, Vol. 132, Article number 052402. DOI: $10.1115 / 1.4000447$

[7] Peraud, J.-P.M., and Hadjiconstantinou, N.G., (2011) "Efficient simulation of multidimensional phonon transport using energy-based variance-reduced Monte Carlo formulations," Physical Review B, Vol. 84, Art. No. 205331

[8] Escobar, R.A., Ghai, S.S., Jhon, M.S., and Amon, C.H., (2006), "Multi-length and time scale thermal transport using the lattice Boltzmann method with applications to electronics cooling," International Journal of Heat and Mass Transfer, Vol. 49, 97107.

[9] Nabovati, A., Sellan, D.P., and Amon, C.A., (2011), "On the Lattice Boltzmann Method for Phonon Transport," Journal of Computational Physics, Vol. 230, pp. 5864-5876.

[10] Murthy, J.Y., and Mathur, S.R., (2002), "Computation of Sub-Micron Thermal Transport Using an Unstructured Finite-Volume Method," Journal of Heat Transfer, Vol. 124, pp. 1176-1181

[11] Narumanchi, S.V.J., (2003), "Simulation of heat transport in sub-micron conduction", PhD. Dissertation, Department of Mechanical Engineering, Carnegie Mellon University.

[12] Narumanchi, S.V.J., Murthy, J.Y., Amon, C.H., (2004), "Sub-micron heat transport model in silicon accounting for phonon dispersion and polarization," Journal of Heat Transfer, Vol. 126, pp. 946-955

[13] Murthy, J.Y., Narumanchi, S.V.J., Pascual-Gutierrez, J.A., Wang, T., Ni, C. and Mathur, S.R., (2005) "Review of Multi-Scale Simulation in Sub-Micron Heat Transfer", International Journal for Multiscale Computational Engineering, Vol. 3, pp. 5-32

[14] Raithby, G.D., and Chui, E.H., (1990), "A Finite-Volume Method for Predicting a Radiant Heat Transfer in Enclosures with Participating Media," Journal of Heat Transfer, Vol. 112(2), pp. 415-423. DOI: 10.1115/1.2910394 
[15] Chai, J.C., Lee, H.S. and Patankar, S.V., (1994), "Finite-Volume Method for Radiative Heat Transfer," Journal of Thermophysics and Heat Transfer, Vol. 8, pp. 419-425, DOI: $10.2514 / 3.559$

[16] Chai, J.C., Lee, H.S., and Patankar, S.V., (1993), "Ray effect and false scattering in the discrete ordinates method," Numerical Heat Transfer, Part B, Vol. 24, pp. 373389, DOI: 10.1080/10407799308955899

[17] Mittal, A., and Mazumder, S., (2011), "Generalized Ballistic-Diffusive Formulation and Hybrid $S_{N}-P_{N}$ Solution of the Boltzmann Transport Equation for Phonons for Non-Equilibrium Heat Conduction," Journal of Heat Transfer, Vol. 133(9), Article No. 092402, DOI: 10.1115/1.4003961

[18] Mittal, A., and Mazumder, S., (2011), "Hybrid Discrete Ordinates-Spherical Harmonics Solution to the Boltzmann Transport Equation for Phonons for NonEquilibrium Heat Conduction," Journal of Computational Physics, Vol. 230(18), pp. 6977-7001, DOI: 10.1016/j.jcp.2011.05.024

[19] Ni, C., and Murthy, J.Y., (2009), "Parallel Computation of the Phonon Boltzmann Transport Equation," Numerical Heat Transfer, Part B, Vol. 55, pp. 435-456.

DOI: 10.1080/10407780902864771

[20] Ali, S.A., Kollu, G., Mazumder, S., Sadayappan, P., and Mittal, A., (2014), "LargeScale Parallel Computation of the Phonon Boltzmann Transport Equation," International Journal of Thermal Sciences, Vol. 86, pp. 341-351.

DOI: 10.1016/j.ijthermalsci.2014.07.019

[21] Chen, G., (2001), "Ballistic-Diffusive Heat Conduction Equations," Phys. Rev. Lett., Vol. 86, pp. 2297-2230.

[22] Chen, G., (2002), "Ballistic-Diffusive Equations for Transient Heat Conduction from Nano to Macroscales," Journal of Heat Transfer, Vol. 124, pp. 320-328.

[23] Yang, R., Chen, G., Laroche, M., and Taur, Y., (2005), "Simulation of Nanoscale Multidimensional Transient Heat Conduction Problems Using Ballistic-Diffusive Equations and Phonon Boltzmann Equation," Journal of Heat Transfer, Vol. 127, pp. 298-306.

[24] Olfe, D.B., (1967), "A modification of the differential approximation for radiative transfer," AIAA Journal, Vol. 5(4), pp. 638-643

[25] Ravishankar, M., Mazumder, S., and Sankar, M., (2010), "Application of the Modified Differential Approximation for Radiative Transfer to Arbitrary Geometry," Journal of Quantitative Spectroscopy and Radiative Transfer, Vol. 111, pp. 2052-2069.

[26] Loy, J.M., Murthy, J.Y., and Singh, D., (2012), “A Fast Hybrid Fourier-Boltzmann Transport Equation Solver for Non-Gray Phonon Transport," Journal of Heat Transfer, Vol. 135, Article Number 011008.

[27] Modest M.F., (2013), Radiative Heat Transfer, Third Edition, Academic Press.

[28] Majumdar, A., (1993), "Microscale Heat Transfer in Dielectric Thin Films," Journal of Heat Transfer, Vol. 115, pp. 7-16. DOI: 10.1115/1.2910673

[29] Whitaker, S., (1983), Fundamental Principles of Heat Transfer, Krieger Publishing Company.

[30] Mazumder, S., (2015), Numerical Methods for Partial Differential Equations: Finite Difference and Finite Volume Methods, First Edition, Academic Press, New York. 
[31] Saad, Y., (2003), Iterative Methods for Sparse Linear Systems, $2^{\text {nd }}$ Edition, SIAM, Philadelphia, PA.

[32] Holland, M.G., (1963), "Analysis of lattice thermal conductivity," Physical Review, Vol. 132(6), pp. 2461-2471. 10.1103/PhysRev.132.2461

[33] Brockhouse, B. N., (1959), "Lattice Vibrations in Silicon and Germanium", Phys. Rev. Lett., Volume 2(6), pp. 256-258. DOI: 10.1103/PhysRevLett.2.256

[34] Peraud, J.-P.M., and Hadjiconstantinou, N.G., (2016), "Extending the range of validity of Fourier's law into the kinetic transport regime via asymptotic solution of the phonon Boltzmann transport equation," Physical Review B, Vol. 93, p. 045424. 


\section{NOMENCLATURE}

$D \quad$ density of states per unit volume $\left[\mathrm{m}^{-3}\right]$

$f \quad$ number density function

$f_{0} \quad$ equilibrium number density function

$G_{\omega, p} \quad$ spectral directionally integrated intensity $\left[\mathrm{Wm}^{-2} \mathrm{rad}^{-1} \mathrm{~s}\right]$

$\hbar \quad$ Dirac (or reduced Planck) constant $=1.0546 \times 10^{-34}\left[\mathrm{~m}^{2} \mathrm{~kg} \cdot \mathrm{s}^{-1}\right]$

$I_{\omega, p} \quad$ spectral directional phonon intensity $\left[\mathrm{Wm}^{-2} \mathrm{sr}^{-1} \mathrm{rad}^{-1} \mathrm{~s}\right]$

$I_{0, \omega, p} \quad$ equilibrium phonon intensity $\left[\mathrm{Wm}^{-2} \mathrm{sr}^{-1} \mathrm{rad}^{-1} \mathrm{~s}\right]$

Kn Knudsen number, spectral or overall

$K n_{c} \quad$ cutoff Knudsen number

$k_{B} \quad$ Boltzmann constant $=1.381 \times 10^{-23}\left[\mathrm{~m}^{2} \mathrm{~kg} \cdot \mathrm{s}^{-2} \mathrm{~K}^{-1}\right]$

$\hat{\mathbf{n}} \quad$ unit surface normal vector

$N_{\text {band }} \quad$ total number of spectral intervals (or bands)

$N_{\text {band }, K n<K n_{c}} \quad$ number of bands for which the Knudsen number is below the cutoff

$N_{\text {cell }} \quad$ number of control volumes (or cells)

$N_{d i r} \quad$ number of control angles (or directions)

$p \quad$ phonon polarization index

q heat flux vector $\left[\mathrm{Wm}^{-2}\right]$

$\mathbf{r} \quad$ position vector $[\mathrm{m}]$

$\hat{\mathbf{s}} \quad$ unit direction vector

$t \quad$ time [s]

$T \quad$ absolute temperature [K]

$u \quad$ internal energy per unit volume $\left[\mathrm{J} / \mathrm{m}^{3}\right]$

$V_{k} \quad$ volume of cell $k\left[\mathrm{~m}^{3}\right]$

\section{Greek}

$\alpha$

$\theta$

$\boldsymbol{v}_{\omega, p}$

$\tau_{\omega, p}$

$\omega$

$\Omega$

$\psi$ degree of specularity

polar angle [radians]

phonon group velocity vector $[\mathrm{m} / \mathrm{s}]$

relaxation time scale [s]

angular frequency $[\mathrm{rad} / \mathrm{s}]$

solid angle (sr)

azimuthal angle [radians] 


\section{List of Figures}

Figure 1: Overall solution algorithm for the hybrid method.

Figure 2: Unstructured stencil showing geometric connectivity and a discrete direction (line of sight) of propagation of phonons.

Figure 3: Geometry and boundary conditions used for the $2 D$ test case. In this study, $L=$ 1 um was used.

Figure 4: Spectral Knudsen numbers for acoustic phonons in silicon computed at two different temperatures, using relaxation time-scales from Ref. [32]. For reference, the cutoff Knudsen number is also shown.

Figure 5: Comparison of the non-dimensional heat fluxes predicted by the three methods for Case 1 (205-195K): (a) bottom wall, and (b) top wall.

Figure 6: Error (compared to full BTE) in center line (C.L.) temperature incurred by the two approximate methods for Case 1.

Figure 7: Steady state temperature distributions obtained using the various methods for a $3 D$ nanostructure: (a) geometry and mesh, (b) CADOM solution, (c) P1 solution, and (d) Hybrid solution.

Figure 8: Non-dimensional wall heat fluxes (normal to wall) computed using the three methods at two different instances of time: (a) top wall at $t=\tau_{\max } / 100$, (b) bottom wall at $t=\tau_{\max } / 100,(c)$ top wall at $t=\tau_{\max } / 10$, and $(d)$ bottom wall at $t=\tau_{\max } / 10$.

Figure 9: Temporal evolution of the temperature distribution computed using the hybrid method with a cutoff Knudsen number of 0.1.

\section{$\underline{\text { List of Tables }}$}

Table 1: Errors incurred in using the $P_{1}$ approximation for solution of the steady state $1 D$ BTE at various Knudsen numbers. The fluxes shown are non-dimensional fluxes.

Table 2: Summary of results of steady state calculations using the various methods. 


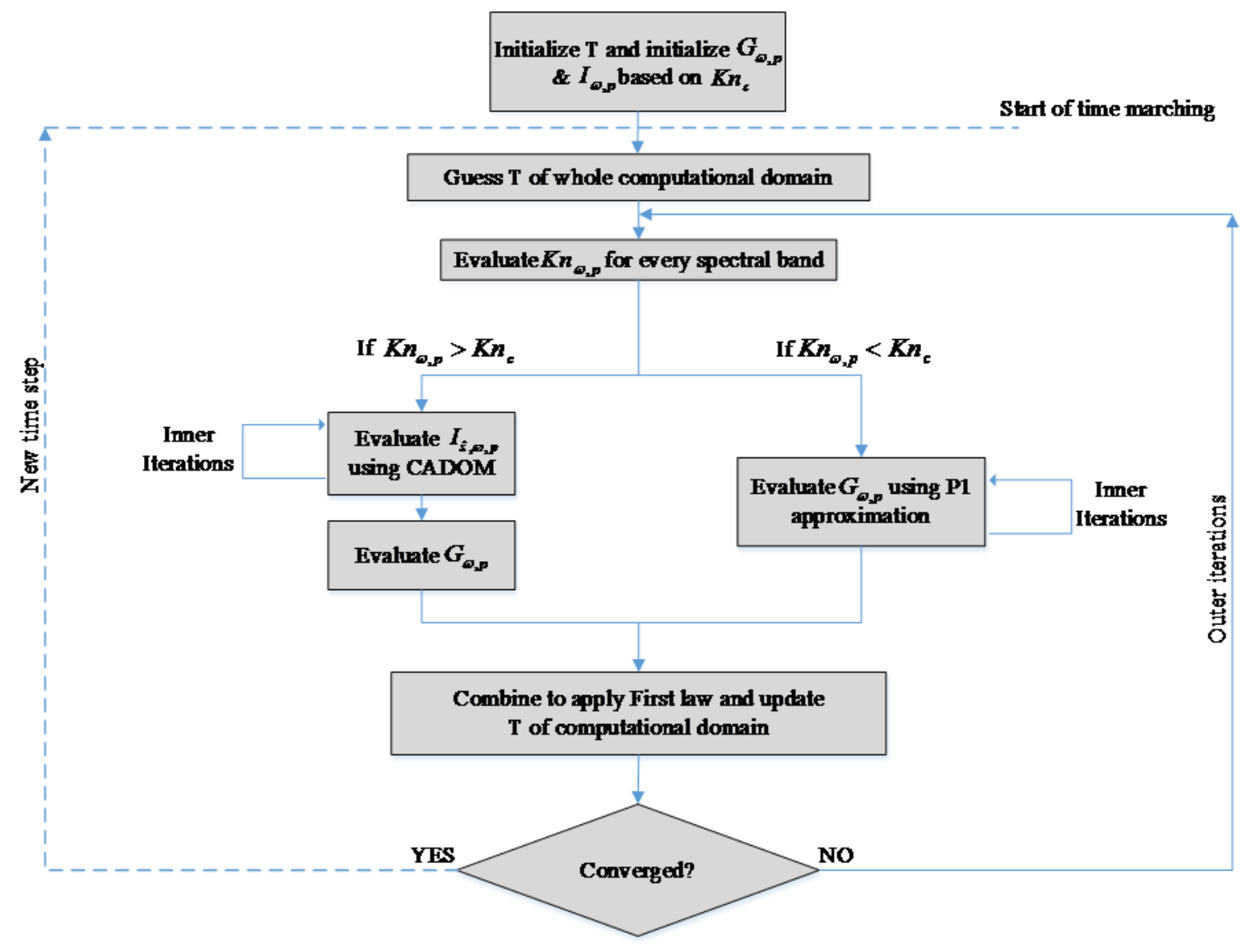

Figure 1: Overall solution algorithm for the hybrid method. 


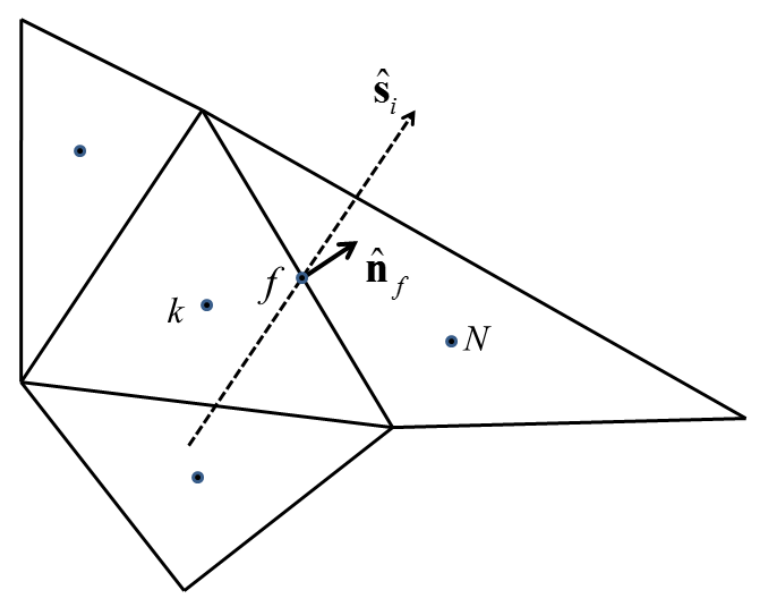

Figure 2: Unstructured stencil showing geometric connectivity and a discrete direction (line of sight) of propagation of phonons. 


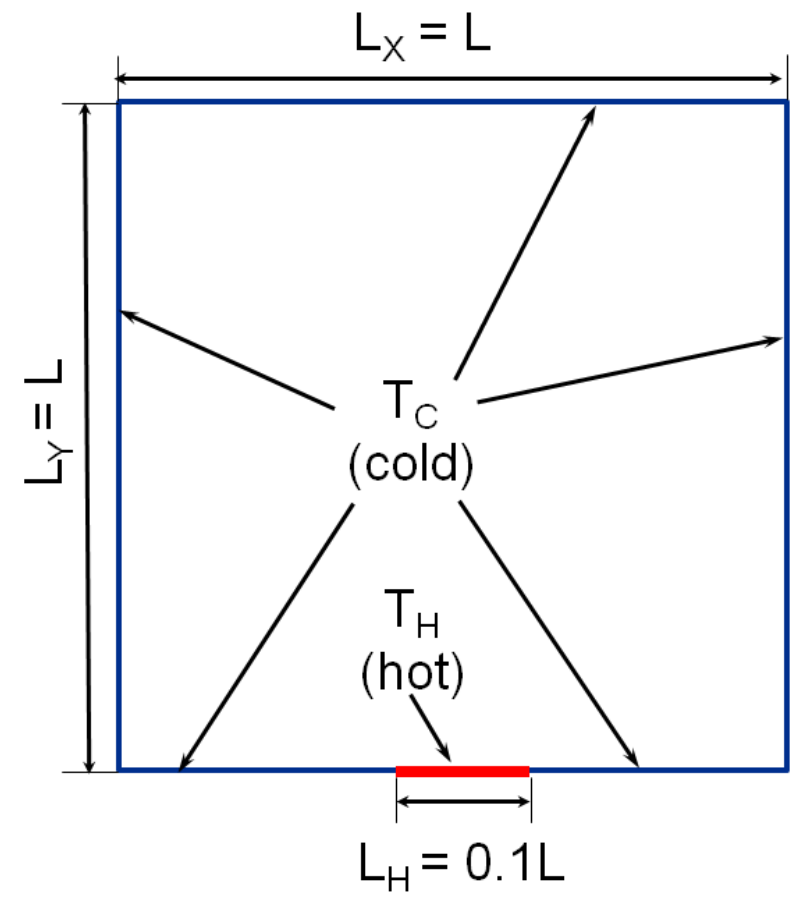

Figure 3: Geometry and boundary conditions used for the $2 D$ test case. In this study, $L=$ 1 um was used. 


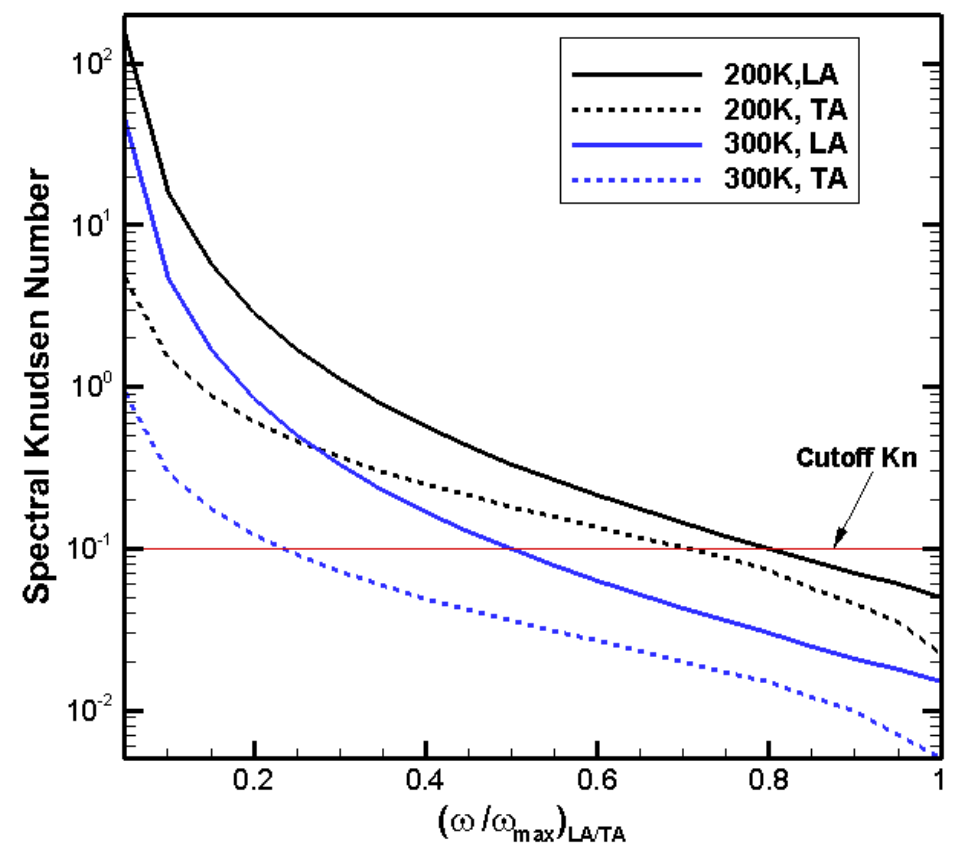

Figure 4: Spectral Knudsen numbers for acoustic phonons in silicon computed at two different temperatures, using relaxation time-scales from Ref. [32]. For reference, the cutoff Knudsen number is also shown. 


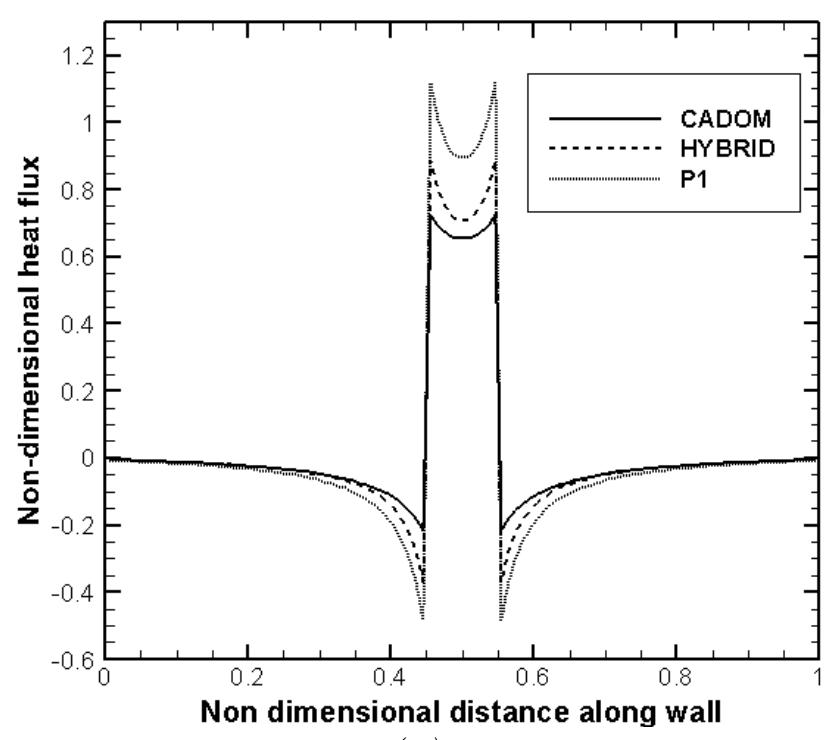

(a)

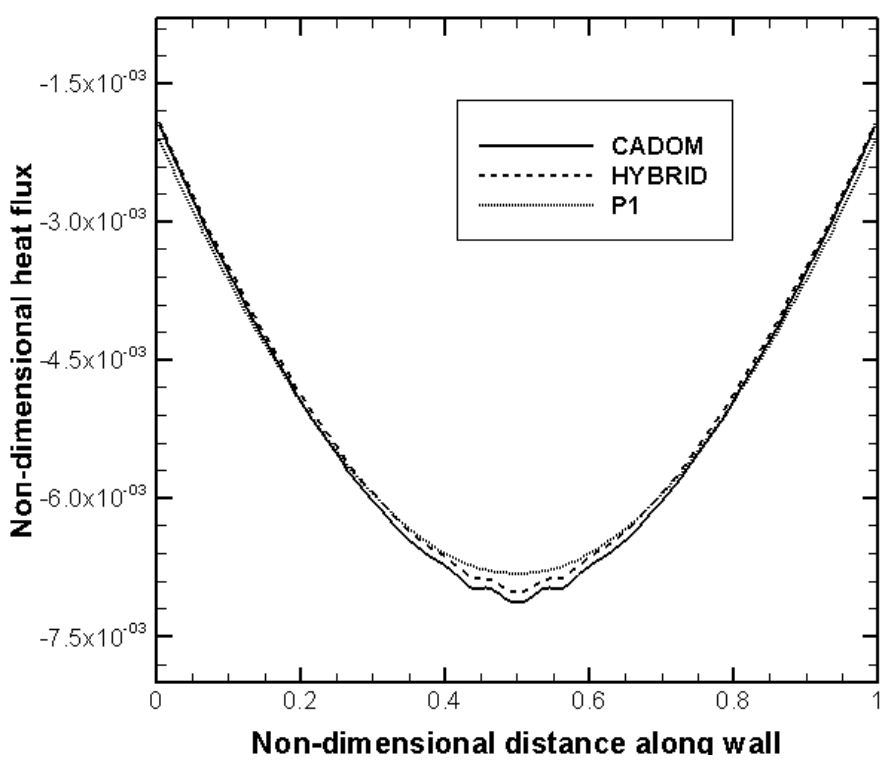

(b)

Figure 5: Comparison of the non-dimensional heat fluxes predicted by the three methods for Case 1 (205-195K): (a) bottom wall, and (b) top wall. 


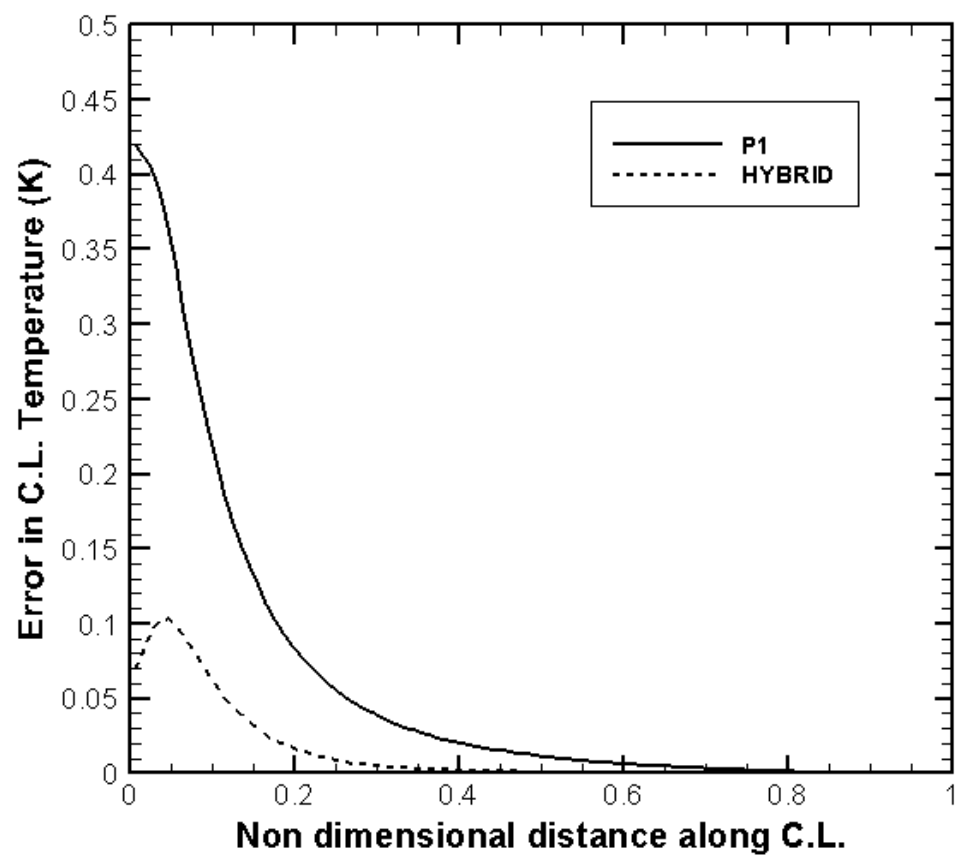

Figure 6: Error (compared to full BTE) in center line (C.L.) temperature incurred by the two approximate methods for Case 1. 


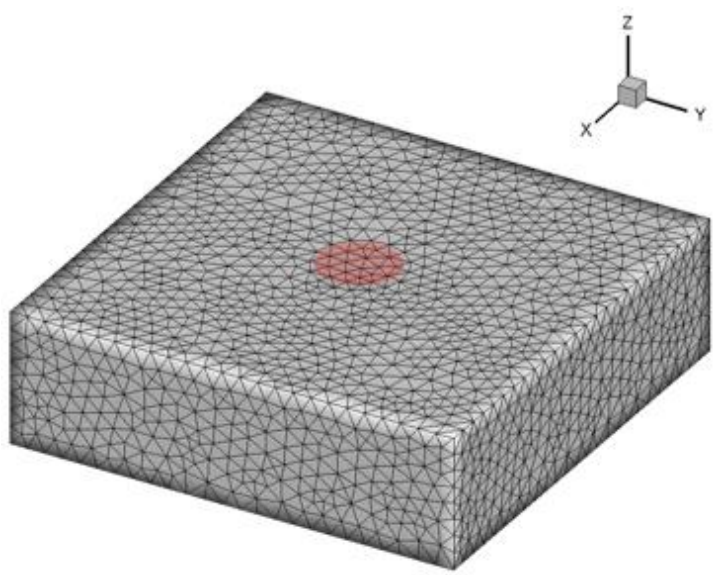

(a)
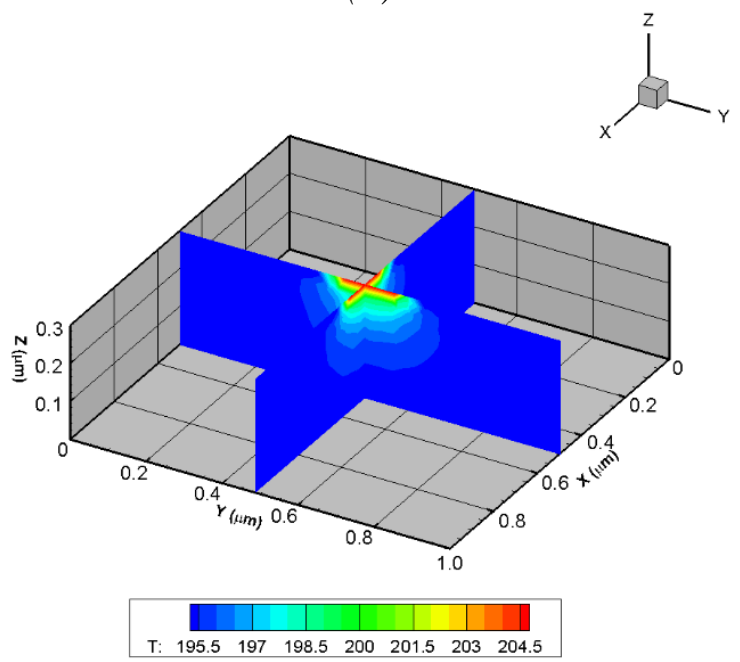

(c)

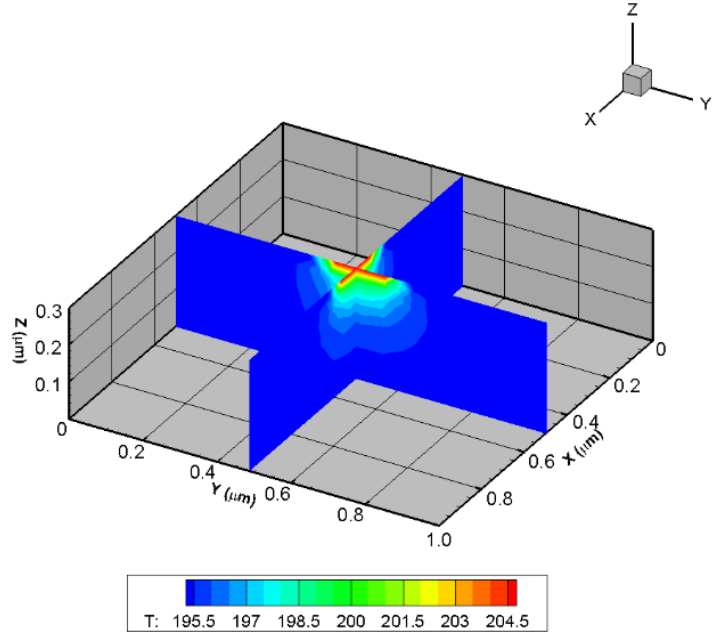

(b)
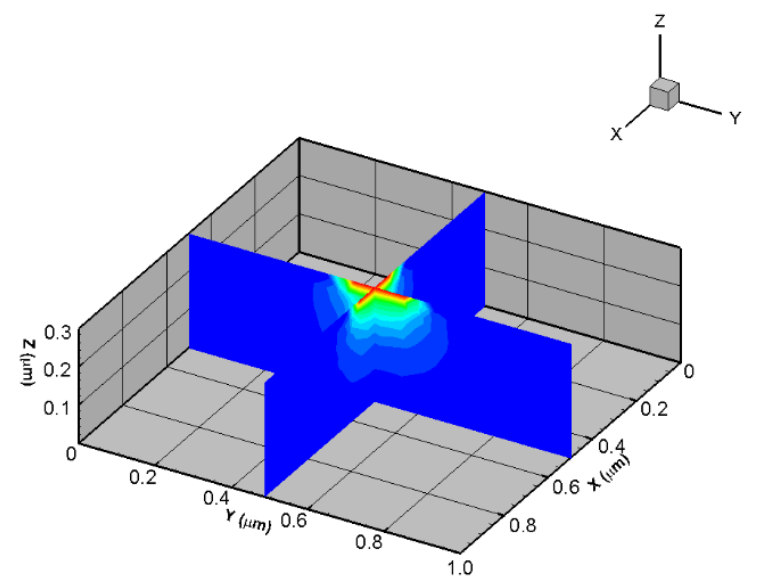

T: $\begin{array}{rrrrrrr}195.5 & 197 & 198.5 & 200 & 201.5 & 203 & 204.5\end{array}$

(d)

Figure 7: Steady state temperature distributions obtained using the various methods for a 3D nanostructure: (a) geometry and mesh, (b) CADOM solution, (c) P1 solution, and (d) Hybrid solution. 

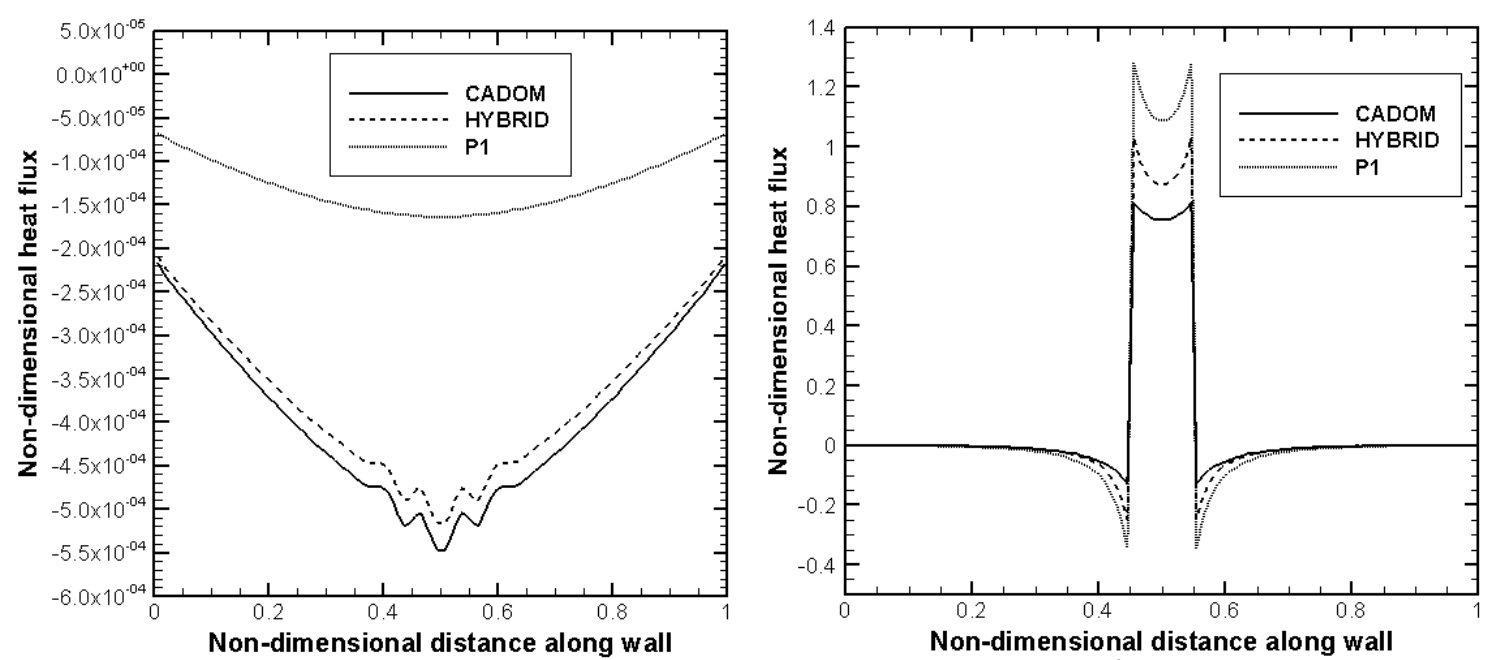

(a)

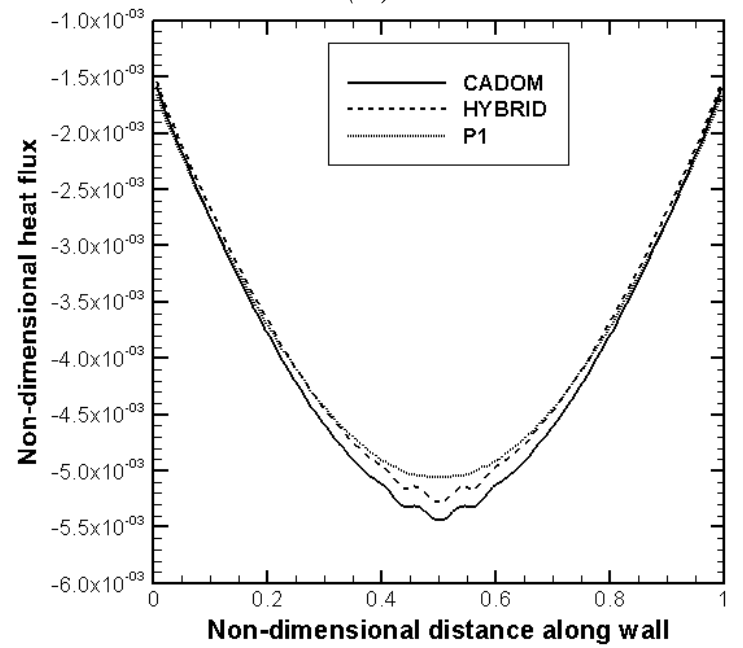

(c)

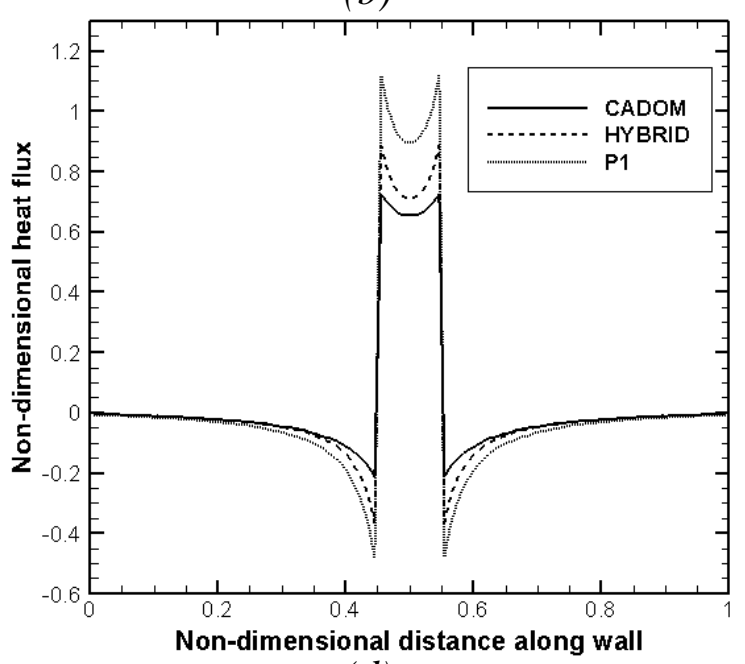

(d)

Figure 8: Non-dimensional wall heat fluxes (normal to wall) computed using the three methods at two different instances of time: (a) top wall at $t=\tau_{\max } / 100$, (b) bottom wall at $t=\tau_{\max } / 100,(c)$ top wall at $t=\tau_{\max } / 10$, and $(d)$ bottom wall at $t=\tau_{\max } / 10$. 

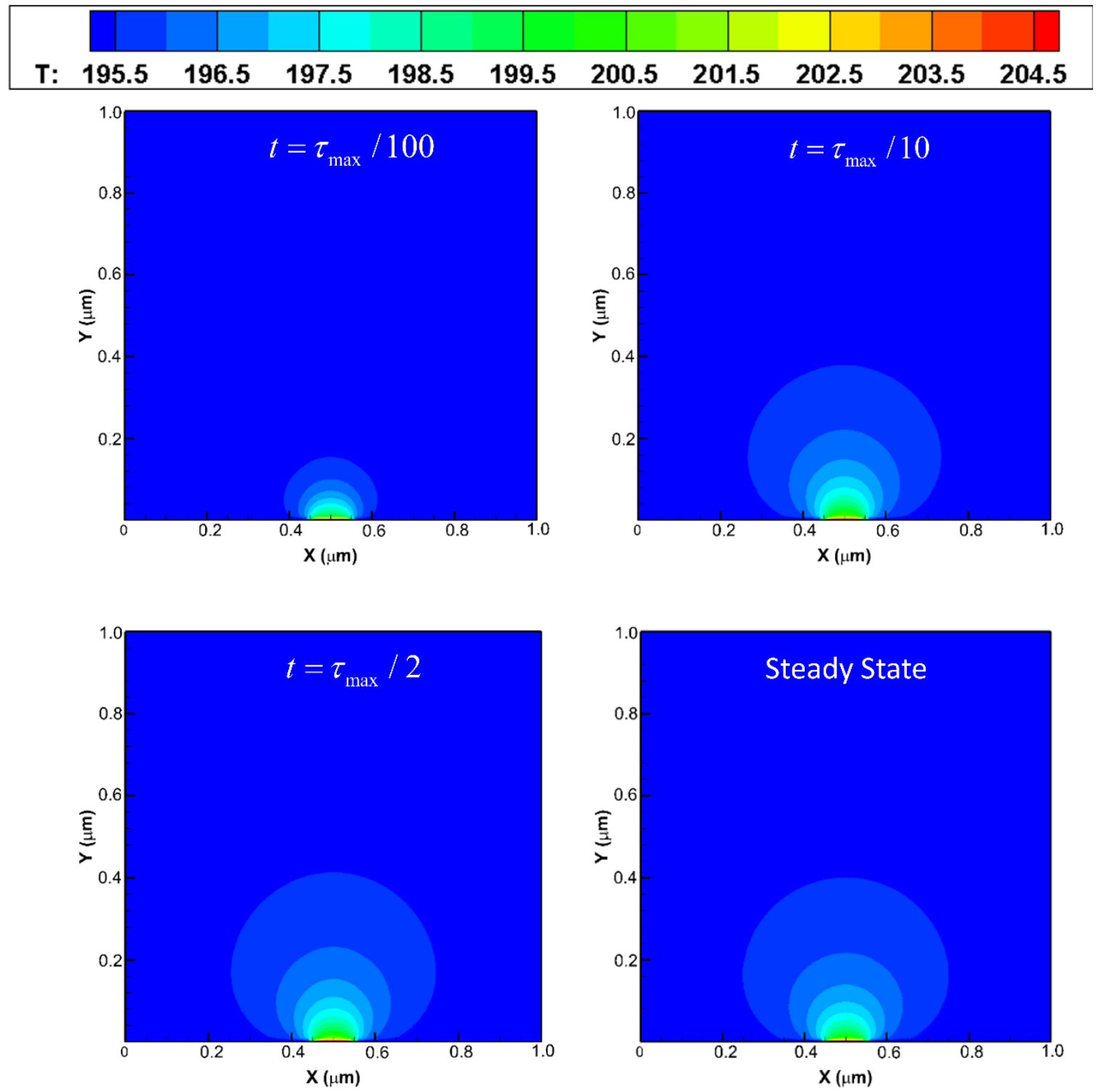

Figure 9: Temporal evolution of the temperature distribution computed using the hybrid method with a cutoff Knudsen number of 0.1. 
Table 1: Errors incurred in using the $P_{1}$ approximation for solution of the steady state ID BTE at various Knudsen numbers. The fluxes shown are non-dimensional fluxes.

\begin{tabular}{|c|c|c|c|c|}
\hline \multirow{2}{*}{$K n$} & \multicolumn{2}{|c|}{ Heat Flux (non-dimensional) } & \multirow{2}{*}{$\begin{array}{c}\text { Error in } \\
\text { Heat Flux }\end{array}$} & $\begin{array}{c}\text { \% Error } \\
\text { (L Norm) in } \\
\text { Temperature }\end{array}$ \\
\cline { 2 - 3 } & Exact BTE & P1 & & 0.0018947 \\
\hline 10.00 & 0.9157 & 0.9302 & 1.5867 & 0.0023375 \\
\hline 5.00 & 0.8492 & 0.8696 & 2.4007 & 0.0024556 \\
\hline 3.00 & 0.7769 & 0.8000 & 2.9731 & 0.0021507 \\
\hline 1.50 & 0.6450 & 0.6667 & 3.3657 & 0.0017378 \\
\hline 1.00 & 0.5533 & 0.5714 & 3.2854 & 0.0009148 \\
\hline 0.50 & 0.3901 & 0.4000 & 2.5455 & 0.0006921 \\
\hline 0.40 & 0.3402 & 0.3478 & 2.2540 & 0.0005786 \\
\hline 0.35 & 0.3117 & 0.3182 & 2.0751 & 0.0004680 \\
\hline 0.30 & 0.2805 & 0.2857 & 1.8734 & 0.0003655 \\
\hline 0.25 & 0.2459 & 0.2500 & 1.6469 & 0.0002775 \\
\hline 0.20 & 0.2076 & 0.2105 & 1.3935 & 0.0002091 \\
\hline 0.15 & 0.1648 & 0.1667 & 1.1107 & 0.0001553 \\
\hline 0.10 & 0.1167 & 0.1176 & 0.7919 & 0.0000959 \\
\hline 0.05 & 0.0622 & 0.0625 & 0.4823 & 0.0000226 \\
\hline 0.01 & 0.0131 & 0.0131 & 0.0913 & \\
\hline
\end{tabular}


Table 2: Summary of results of steady state calculations using the various methods.

\begin{tabular}{|c|c|c|c|c|c|c|}
\hline \multirow[t]{2}{*}{ Test Case } & \multirow[t]{2}{*}{$K n_{c}$} & \multirow[t]{2}{*}{ Method } & \multirow[t]{2}{*}{$\begin{array}{l}\text { Bands for } \\
\text { Hybrid }\end{array}$} & \multirow[t]{2}{*}{ CPU Time } & \multicolumn{2}{|c|}{$\begin{array}{c}\text { \% Error in Heat } \\
\text { Flux }\end{array}$} \\
\hline & & & & & Top & Bottom \\
\hline \multirow{5}{*}{$\begin{array}{c}\text { Case } 1 \\
(205-195 K)\end{array}$} & 0.05 & Hybrid & $\begin{array}{c}37 \text { CADOM, } \\
3 \mathrm{P}_{1}\end{array}$ & $9 \mathrm{hrs} 12 \mathrm{~m}$ & 0.73 & 1.78 \\
\hline & \multirow{3}{*}{0.1} & CADOM & \multirow{3}{*}{$\begin{array}{c}30 \text { CADOM, } \\
10 \mathrm{P}_{1}\end{array}$} & $9 \mathrm{hrs} 42 \mathrm{~m}$ & - & - \\
\hline & & $\mathrm{P} 1$ & & $2 \mathrm{hrs} 27 \mathrm{~m}$ & 7.89 & 48.65 \\
\hline & & Hybrid & & $8 \mathrm{hrs} 36 \mathrm{~m}$ & 1.53 & 13.50 \\
\hline & 0.2 & Hybrid & $\begin{array}{c}21 \mathrm{CADOM}, \\
19 \mathrm{P}_{1}\end{array}$ & $5 \mathrm{hrs} 42 \mathrm{~m}$ & 1.39 & 27.92 \\
\hline \multirow{5}{*}{$\begin{array}{c}\text { Case } 2 \\
(255-245 K)\end{array}$} & 0.05 & Hybrid & $\begin{array}{c}28 \text { CADOM, } \\
12 \mathrm{P}_{1}\end{array}$ & $20 \mathrm{hrs} 51 \mathrm{~m}$ & 4.78 & 4.85 \\
\hline & \multirow{3}{*}{0.1} & CADOM & \multirow{3}{*}{$\begin{array}{c}20 \mathrm{CADOM} \mathrm{C} \\
20 \mathrm{P}_{1}\end{array}$} & $26 \mathrm{hrs} 58 \mathrm{~m}$ & - & - \\
\hline & & $\mathrm{P} 1$ & & $3 \mathrm{hrs} 33 \mathrm{~m}$ & 4.52 & 25.61 \\
\hline & & Hybrid & & $16 \mathrm{hrs} 42 \mathrm{~m}$ & 5.65 & 12.32 \\
\hline & 0.2 & Hybrid & $\begin{array}{c}12 \text { CADOM, } \\
28 \mathrm{P}_{1}\end{array}$ & $11 \mathrm{hrs} 35 \mathrm{~m}$ & 5.52 & 18.83 \\
\hline \multirow{5}{*}{$\begin{array}{c}\text { Case } 3 \\
(305-295 \mathrm{~K})\end{array}$} & 0.05 & Hybrid & $\begin{array}{c}20 \text { CADOM, } \\
20 \mathrm{P}_{1}\end{array}$ & $38 \mathrm{hrs} 30 \mathrm{~m}$ & 11.21 & 7.28 \\
\hline & \multirow{3}{*}{0.1} & CADOM & \multirow{3}{*}{$\begin{array}{c}13 \text { CADOM, } \\
27 \mathrm{P}_{1}\end{array}$} & $60 \mathrm{hrs} 18 \mathrm{~m}$ & - & - \\
\hline & & $\mathrm{P} 1$ & & $6 \mathrm{hrs} 38 \mathrm{~m}$ & 10.84 & 9.14 \\
\hline & & Hybrid & & $27 \mathrm{hrs} 55 \mathrm{~m}$ & 11.90 & 6.63 \\
\hline & 0.2 & Hybrid & $\begin{array}{c}9 \mathrm{CADOM}, \\
31 \mathrm{P}_{1}\end{array}$ & $20 \mathrm{hrs} 59 \mathrm{~m}$ & 11.80 & 6.90 \\
\hline
\end{tabular}

